\title{
What is the evidence that tau pathology spreads through prion-like propagation?
}

\author{
Amrit Mudher ${ }^{1 *}$, Morvane Colin², Simon Dujardin ${ }^{3}$, Miguel Medina ${ }^{4}$, Ilse Dewachter ${ }^{5}$, Seyedeh Maryam Alavi Naini ${ }^{6}$,
} Eva-Maria Mandelkow ${ }^{7,8,9}$, Eckhard Mandelkow ${ }^{7,8,9}$, Luc Buée ${ }^{2}$, Michel Goedert ${ }^{10}$ and Jean-Pierre Brion ${ }^{11^{*}}$

\begin{abstract}
Emerging experimental evidence suggests that the spread of tau pathology in the brain in Tauopathies reflects the propagation of abnormal tau species along neuroanatomically connected brain areas. This propagation could occur through a "prion-like" mechanism involving transfer of abnormal tau seeds from a "donor cell" to a "recipient cell" and recruitment of normal tau in the latter to generate new tau seeds. This review critically appraises the evidence that the spread of tau pathology occurs via such a "prion-like" mechanism and proposes a number of recommendations for directing future research. Recommendations for definitions of frequently used terms in the tau field are presented in an attempt to clarify and standardize interpretation of research findings. Molecular and cellular factors affecting tau aggregation are briefly reviewed, as are potential contributions of physiological and pathological post-translational modifications of tau. Additionally, the experimental evidence for tau seeding and "prion-like" propagation of tau aggregation that has emerged from cellular assays and in vivo models is discussed. Propagation of tau pathology using "prion-like" mechanisms is expected to incorporate several steps including cellular uptake, templated seeding, secretion and intercellular transfer through synaptic and non-synaptic pathways. The experimental findings supporting each of these steps are reviewed. The clinical validity of these experimental findings is then debated by considering the supportive or contradictory findings from patient samples. Further, the role of physiological tau release in this scenario is examined because emerging data shows that tau is secreted but the physiological function (if any) of this secretion in the context of propagation of pathological tau seeds is unclear. Bona fide prions exhibit specific properties, including transmission from cell to cell, tissue to tissue and organism to organism. The propagation of tau pathology has so far not been shown to exhibit all of these steps and how this influences the debate of whether or not abnormal tau species can propagate in a "prion-like" manner is discussed. The exact nature of tau seeds responsible for propagation of tau pathology in human tauopathies remains controversial; it might be tightly linked to the existence of tau strains stably propagating peculiar patterns of neuropathological lesions, corresponding to the different patterns seen in human tauopathies. That this is a property shared by all seed-competent tau conformers is not yet firmly established. Further investigation is also required to clarify the relationship between propagation of tau aggregates and tau-induced toxicity. Genetic variants identified as risks factors for tauopathies might play a role in propagation of tau pathology, but many more studies are needed to document this. The contribution of selective vulnerability of neuronal populations, as an alternative to prion-like mechanisms to explain spreading of tau pathology needs to be clarified. Learning from the prion field will be helpful to enhance our understanding of propagation of tau pathology. Finally, development of better models is expected to answer some of these key questions and allow for the testing of propagation-centred therapies.
\end{abstract}

Keywords: Alzheimer's disease, tau, prion-like propagation, transmission, tauopathies, aggregation, seeding

\footnotetext{
* Correspondence: A.Mudher@soton.ac.uk; jpbrion@ulb.ac.be

'University of Southampton, Biological Sciences, Faculty of Natural and

Environmental Sciences, SO17 1BJ Southampton, UK

${ }^{11}$ Laboratory of Histology, Neuroanatomy and Neuropathology Université

Libre de Bruxelles, Faculty of Medicine, ULB Neuroscience Institute (UNI) 808,

route de Lennik 1070, Brussels, Belgium

Full list of author information is available at the end of the article
}

(c) The Author(s). 2017 Open Access This article is distributed under the terms of the Creative Commons Attribution 4.0 International License (http://creativecommons.org/licenses/by/4.0/), which permits unrestricted use, distribution, and reproduction in any medium, provided you give appropriate credit to the original author(s) and the source, provide a link to the Creative Commons license, and indicate if changes were made. The Creative Commons Public Domain Dedication waiver (http://creativecommons.org/publicdomain/zero/1.0/) applies to the data made available in this article, unless otherwise stated. 


\section{Introduction}

The sequential appearance of tau pathology in the brains of Tauopathy patients has traditionally been considered to arise due to differential vulnerability of susceptible brain regions to disease processes, which is then reflected in the stereotypical progression of lesions throughout the brain. Recent evidence challenges this view and promotes the idea that tau pathology spreads through the brain using a prion-like mechanism. During the first EUROTAU meeting (Lille, France, April 2017 (http://lucbuee.fr/crbst_10.html), a round table discussion critically appraised this evidence and reflected on its clinical relevance. This review summarises that debate and makes recommendations that were suggested for clarification and identification of key-points for future studies. Additionally it was noted that various terms are used to describe tau pathology and that this can lead to confusion. Defining these terms would clarify their meaning and therefore standardise their use in future publications.

\section{Key definitions}

There are numerous terms that are commonly used to describe aspects of tau pathology. These are listed and defined in Table 1 together with recommendations for consistent usage in future publications.

\section{Round table discussion and questions for tau research Tau aggregation \\ Mechanisms of tau aggregation}

Six tau isoforms are expressed in adult human brain, differing by the presence of 0,1 , or 2 amino-terminal inserts, and the inclusion or not of an amino acid repeat in the carboxy-terminal half [57]. Amino-terminal inserts are encoded by exons 2 and 3, with exon 3 never being expressed without exon 2, and the carboxy-terminal insert is encoded by exon 10. Assembled tau proteins are the molecular components of neurofibrillary tangles found in AD [18]. The assembly of monomeric tau into higherorder molecular species leads to the formation of tau filaments composing these neurofibrillary tangles. This ordered assembly must underlie tau seeding and recruitment of normal tau by pathological tau species to form aggregates made of filaments. Progressive formation of these filamentous tau aggregates is associated with insolubility (a biochemical definition) but insoluble tau may or may not be composed of filaments. However this information is not always provided in publications. These aggregates form different types of tau inclusions: e.g. neurofibrillary tangles and neuropil threads in AD and other tauopathies, argyrophilic grains in argyrophilic grain disease (AGD), oligodendroglial coiled bodies in tauopathies, astrocytic tufts in progressive supranuclear palsy (PSP), astrocytic plaques in corticobasal degeneration (CBD), Pick bodies in Pick disease, etc. (for review see $[9,85]$ ).

A lot of information is now available about which domains are needed for assembly of tau into filaments, and about the molecular arrangement of tau into paired helical (PHFs) and straight filaments (SFs). Assembly into filaments needs the tau repeats that form part of the core of, with the $\mathrm{N}$-terminal half and the C-terminus extending outside to form the "fuzzy coat" [56, 139]. Hexapeptides in repeats 2 and 3 are needed for the induced aggregation of recombinant tau into filaments [134]. High-resolution structures of tau filaments from $\mathrm{AD}$ brain have been determined by cryogenic electron microscopy. The cores of PHFs and SFs from AD brain consist of two identical protofilaments extending from residues V306 to F378, which are arranged base-to-base and back-to-base, respectively (ultrastructural polymorphs) illustrated in Fig. 1. The protofilaments probably define the seeds for tau aggregation. Each protofilament comprises eight beta-sheets, which adopt a combined crossbeta/beta-helix structure [46].

Oligomeric assembly of tau leads to the formation of soluble, non-filamentous species that are present in the brains of AD patients and some mouse models [88]. The accumulation of these oligomers might precede the formation of tau filaments, but their systematic contribution to the formation of filamentous insoluble aggregates in tauopathies needs further investigation.

The term "aggregation" is often meant to imply selfassembly of tau molecules into a filamentous form containing beta-pleated sheet structure similar to Alzheimer PHFs and SFs, but this is often not shown. Therefore the build-up of tau in the cell in various models of tauopathy should be described by a more neutral term, such as accumulation, if this information is not available.

What triggers tau seeding and aggregation in neuronal cells in vivo is still unclear. Tau inclusions in tauopathies have been observed to be associated with many other molecules that might play a role in promoting tau aggregation. Recombinant tau assembly can be triggered in vitro by heparin and other sulphated glycosaminoycans, RNA, as well as arachidonic acid [58, 68, 81, 138]. The strongly negative charges on each of these species (heparin, dextran sulphate, arachidonic acid and RNA) are likely to act as critical motifs for interaction with tau in vivo.

Tau may be involved in phase separation, with droplets forming through an interaction between the positively charged microtubule-binding domains and negatively charged molecules [5, 147]. Beta-sheet structures have been detected in these membraneless droplets. However, it remains to be seen what these in vitro findings mean for the aggregation of tau in vivo. The accumulation of non-filamentous tau should not be equated with tau 
Table 1 Terminology for main tau assemblies and definition criteria

\begin{tabular}{|c|c|c|c|}
\hline Name & Definition & Structural criteria & Molecular criteria \\
\hline Tau pathology & $\begin{array}{l}\text { Broad term designing abnormal } \\
\text { molecular changes of normal tau as well as } \\
\text { morphological changes. }\end{array}$ & $\begin{array}{l}\text { Mislocalization of tau and/or pathological tau } \\
\text { assembly in inclusion or aggregate. }\end{array}$ & $\begin{array}{l}\text { Post-translational modifications } \\
\text { of tau. Tau insolubility. }\end{array}$ \\
\hline Tau inclusion & $\begin{array}{l}\text { Morphologically distinct subcellular } \\
\text { structure inside a cell. }\end{array}$ & $\begin{array}{l}\text { Microscopically visible structure. Made of tau } \\
\text { aggregates. }\end{array}$ & Properties of tau aggregates. \\
\hline Tau aggregate & $\begin{array}{l}\text { Assembly of tau into oligomers, } \\
\text { fibrils, filaments, and NFT }\end{array}$ & $\begin{array}{l}\text { Molecular tau assembly based on highly } \\
\text { ordered ß-sheet structure. }\end{array}$ & $\begin{array}{l}\text { Positive with ß-sheet (amyloid) } \\
\text { sensitive dyes (Thioflavine T, } \\
\text { Congo Red, LCOs). Tau } \\
\text { hyperphosphorylation }\end{array}$ \\
\hline Tau seed & A tau species inducing aggregation of tau & $\begin{array}{l}\text { Molecular tau assemblies of various size } \\
\text { providing a template }\end{array}$ & $\begin{array}{l}\text { Positive with B-sheet } \\
\text { sensitive dyes }\end{array}$ \\
\hline $\begin{array}{l}\text { Liquid coacervates } \\
\text { of tau }\end{array}$ & $\begin{array}{l}\text { Membraneless organelles in a state of } \\
\text { Liquid-liquid phase separation }\end{array}$ & Coacervation of tau into liquid droplets & $\begin{array}{l}\text { Can acquire ß-sheet } \\
\text { structure. }\end{array}$ \\
\hline Tangles & Neuronal tau inclusions in somata & $\begin{array}{l}\text { Composed of bundles of PHFs and SFs. } \\
\text { Gallyas and Campbell-Switzer positive. }\end{array}$ & $3 R$ and $4 R$ tau positive in $A D$ \\
\hline Neuropil threads & Tau inclusions in nerve cell dendrites & $\begin{array}{l}\text { Composed of bundles of PHFs and SFs. } \\
\text { Gallyas and Campbell-Switzer positive. }\end{array}$ & $3 R$ and $4 R$ tau positive in $A D$ \\
\hline Dystrophic neurites & $\begin{array}{l}\text { Axons forming the neuritic corona of } \\
\text { plaques }\end{array}$ & $\begin{array}{l}\text { Nerve cell processes in contact with Aß } \\
\text { deposits. Some of them contain PHFs and } \\
\text { SFs and are Gallyas and Campbell-Switzer } \\
\text { positive. }\end{array}$ & $3 R$ and $4 R$ tau positive in $A D$ \\
\hline Argyrophilic grains & $\begin{array}{l}\text { Neuronal granular tau inclusions in } \\
\text { dendrites }\end{array}$ & $\begin{array}{l}\text { Tau filaments. Gallyas positive. } \\
\text { Campbell-Switzer negative. }\end{array}$ & $4 R$ tau positive in $A G D$ \\
\hline Pick bodies & $\begin{array}{l}\text { Spherical tau inclusions in nerve cell } \\
\text { somata }\end{array}$ & $\begin{array}{l}\text { Filamentous and vesicular material. Gallyas- } \\
\text { negative. Campbell-Switzer positive. }\end{array}$ & $\begin{array}{l}\text { 3R tau positive in Pick } \\
\text { disease. }\end{array}$ \\
\hline $\begin{array}{l}\text { Oligodendroglial } \\
\text { coiled bodies }\end{array}$ & $\begin{array}{l}\text { Tau inclusions in cell bodies of } \\
\text { oligodendrocytes }\end{array}$ & $\begin{array}{l}\text { PHF/SF like filaments. Gallyas positive. } \\
\text { Campbell-Switzer negative. }\end{array}$ & $\begin{array}{l}\text { 4R tau positive in PSP and } \\
\text { CBD }\end{array}$ \\
\hline $\begin{array}{l}\text { Globular } \\
\text { oligodendroglial } \\
\text { inclusions }\end{array}$ & Globular oligodendroglial tau inclusion & Gallyas positive. & $\begin{array}{l}\text { Mainly } 4 \mathrm{R} \text { tau positive in } \\
\text { GGTs }\end{array}$ \\
\hline Tufted astrocytes & $\begin{array}{l}\text { Astrocytes with thin and long radial } \\
\text { processes containing tau inclusions }\end{array}$ & $\begin{array}{l}\text { Tau filaments in cytoplasm and proximal } \\
\text { portions of astrocytic processes. Gallyas positive. } \\
\text { Campbell-Switzer negative. }\end{array}$ & 4R tau positive in PSP \\
\hline Astrocytic plaques & $\begin{array}{l}\text { Astrocytes containing tau inclusions in a } \\
\text { corona-like arrangment }\end{array}$ & $\begin{array}{l}\text { Tau filaments in distal portions of astrocytic } \\
\text { processes. Gallyas positive. Campbell-Switzer negative. }\end{array}$ & 4R tau positive in $C B D$ \\
\hline $\begin{array}{l}\text { Thorn-shaped } \\
\text { astrocytes }\end{array}$ & $\begin{array}{l}\text { Astrocytes with thorn-shaped processes } \\
\text { containing tau inclusions }\end{array}$ & $\begin{array}{l}\text { Spine-like perinuclear tau filaments. Gallyas } \\
\text { positive. }\end{array}$ & $4 R$ tau positive in ARTAG \\
\hline
\end{tabular}

AD Alzheimer's disease, AGD Argyrophilic grain disease, ARTAG, Ageing-related tau astrogliopathy, CBD Corticobasal degeneration, GGTs Globular glial tauopathies, PSP Progressive supranuclear palsy, LCOs Luminescent conjugated oligothiophenes

Each of the various tau inclusions is positive with some LCOs

A common feature of all tau assemblies is their immunoreactivity with tau antibodies, although peculiar tau epitopes can distinguish between them. For more details on specific tau inclusions, tauopathies, and silver staining properties see $[9,54,86,131]$

aggregation. Local tau accumulations, not proven to be tau filaments, should not be confounded with tau aggregates. This is in agreement with observations indicating that cell stressors and signalling mechanisms can induce cellular accumulations of tau [61].

The initial transformation of normal monomeric tau into an abnormal tau seed is still a poorly understood event. A spontaneous, energetically favourable, acquired or inherited conformational change is a possibility. The growth of filaments by addition of tau species might rely on different mechanisms such as templated assembly or nucleated seeding. These are discussed in later sections of this review.
The molecular size of the tau assemblies that have the highest seeding efficiency when added to cultured cells, or injected in animal models, is still the subject of investigation. These studies are discussed in later sections of this review.

\section{Assembly of different tau isoforms}

Significant information is available on the relative incorporation of different tau isoforms in tau inclusions in different tauopathies. E.g. 3R and $4 R$ tau isoforms accumulate in NFTs in AD, 4R tau accumulates in tau inclusions in PSP, CBD, AGD and 3R tau accumulates in tau inclusions in Pick disease. MAPT mutations give rise to 


\section{a}

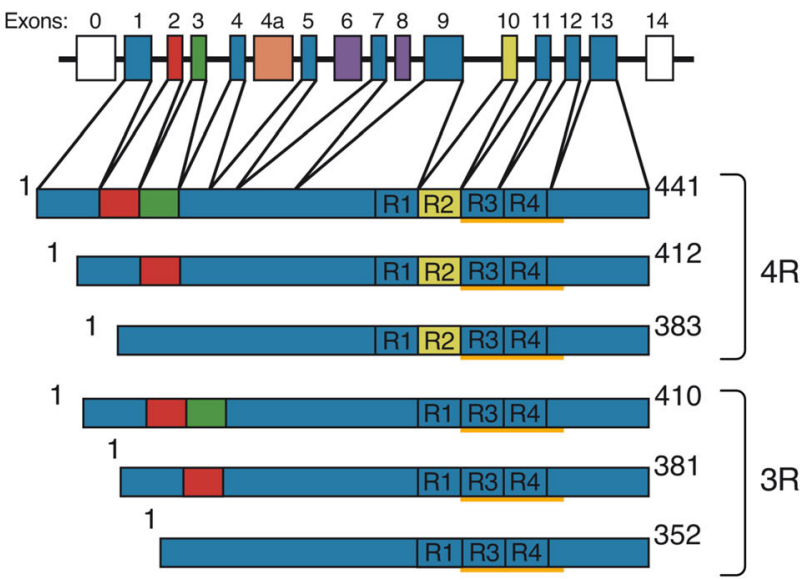

b

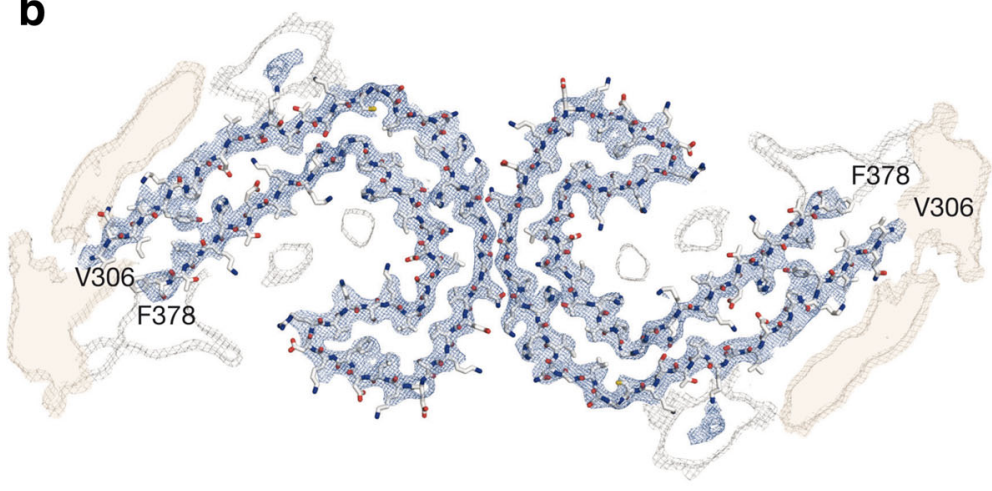

C

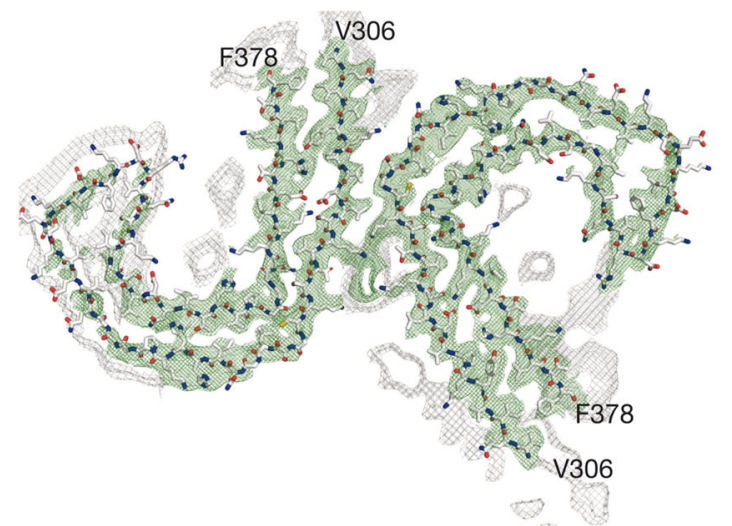

Fig. 1 Human brain tau isoforms and the cores of tau filaments from Alzheimer's disease. a MAPT and the six tau isoforms expressed in adult human brain. MAPT consists of 16 exons (E). Alternative mRNA splicing of E2 (red), E3 (green) and E10 (yellow) gives rise to the six tau isoforms (352-441 amino acids). The constitutively spliced exons (E1, E4, E5, E7, E9, E11, E12 and E13) are shown in blue. E0, which is part of the promoter, and E14 are noncoding (white). E6 and E8 (violet) are not transcribed in human brain. E4a (orange) is expressed only in the peripheral nervous system. The repeats (R1-R4) are shown, with three isoforms having four repeats each (4R) and three isoforms having three repeats each (3R). The core regions of the tau filaments from AD brain (V306-F378, using the numbering of the 441 amino acid tau isoform) are underlined. b, c Cross-sections of the cryogenic electron microscopy (cryo-EM) densities and atomic models of the cores of paired helical (b, in blue) and straight (c, in green) tau filaments. Each filament core consists of two identical protofilaments extending from V306-F378 of tau, which are arranged base-to-base (b) or back-to-base (c). The cryo-EM maps of the filament cores are at 3.4-3.5 ̊ resolution. Unsharpened, $4.5 \AA$ low-pass filtered density is shown in grey. Density highlighted with an orange background is reminiscent of a less-ordered $\beta$-sheet and could accommodate an additional 16 amino acids, which would correspond to a mixture of residues 259-274 (R1) from 3R tau and residues 290-305 (R2) from 4R tau. Adapted from [46] 
tau inclusions made of either $3 \mathrm{R}+4 \mathrm{R}$ tau (V337M, R406W), 3R tau (G272V, deltaK280) or 4R tau (P301L, P301S and all intronic mutations) [35]. Tau filaments have varying morphologies in these inclusions, reflecting (but not always) their tau isoforms composition [19, 55]. In vitro experiments indicate that $4 \mathrm{R}$ tau has a greater aggregation propensity than $3 R$ tau [2], potentially underlying a mechanism by which pathological $4 R$ tau species might assemble preferentially in vivo in $4 R$ tau filaments. In vitro $4 \mathrm{R}$ tau can drive $3 \mathrm{R}$ tau aggregation and the presence of exons 2 and 10 promotes tau aggregation, whereas exon 3 depresses it [149]. 4R and 3R tau isoforms (with different N-terminal inserts) are however both expressed in human tauopathies and why $4 \mathrm{R}$ tau or $3 \mathrm{R}$ tau species start a selective assembly in vivo needs further studies.

In $4 \mathrm{R}$ tauopathies like PSP, AGD and CBD, 4R tau inclusions develop significantly in astrocytes (astrocytic tufts, astrocytic plaques) and in oligodendrocytes (coiled bodies) and the reason for the susceptibility of these glial cells (in addition to neurons) to develop tau aggregates in these tauopathies needs further research.

\section{Role of tau phosphorylation and other posttranslational changes in aggregation}

Tau is a phosphoprotein containing 85 potential serine, threonine and tyrosine phosphorylation sites and tau phosphorylation physiologically modulates microtubule assembly [9]. Soluble tau in biopsy-derived material purified from normal brain is phosphorylated but tau dephosphorylation occurs rapidly post-mortem in autopsyderived material from both human [92, 117] and rodent brains [98]. Tau phosphorylation is affected in several physiological conditions; e.g. foetal tau is more highly phosphorylated than adult tau and tau phosphorylation is higher during hibernation [10] and is increased during hypothermia [104]. In contrast, tau in aggregates in tauopathies is always hyperphosphorylated and this hyperphosphorylation is persistent even after long post-mortem delays.

Various groups have recently established lists of post translational modifications (PTMs) highlighted in different models (murine, human), including the Hanger group (https:/docs.google.com/spreadsheets/d/1hGYs1ZcupmT nbB7n6qs1r_WVTXHt1O7NBLyKBN7EOUQ/edit\#gid=0), the Mucke group [96] and the Kuret Group [47]. A study comparing wild-type mice and transgenic mice with abundant Abeta deposits in the absence of filamentous tau inclusions showed similar PTMs of tau, unlike what is seen for filamentous tau in AD [96]. Additional studies of animal models with filamentous tau inclusions would be informative. Meanwhile, it is possible to analyse the literature by focusing on the PTMs of tau in AD brains that are absent from control brains.
Phosphorylation is undoubtedly the most prominent PTM in AD brains and there are some phosphoepitopes present exclusively in AD brains and not found in normal healthy biopsy brain. For instance, phospho-Ser422 is found in different Tauopathies including AD but not in healthy biopsy-derived brain materials [20,67]. Some antibodies also recognize conformational epitopes specific to tau assemblies. In vitro, phosphorylation is not a prerequisite for tau aggregation since tau assembly can be achieved with recombinant tau in the presence of different fibrillation inducers $[24,58,103]$. Nonetheless it can influence aggregation as was shown recently whereby prephosphorylated recombinant mutant tau proteins were used as an aggregation inducers and enabled the in vitro aggregation of recombinant mutant tau proteins [37]. Furthermore, equivalent tau seeding behaviour has been demonstrated for both nonphosphorylated and phosphorylated P301S recombinant tau proteins generated in vitro [45]. Intracerebral injection of preformed fibrils made of non-phosphorylated recombinant mutant tau enhances and accelerates propagation of tau pathology in mouse models expressing the same tau isoform $[75,102,124]$. Intriguingly though, endogenous tau seeded by these injections then propagates phosphotau epitopes. Hyperphosphorylation of tau might nevertheless play a role in modulating propagation of tau pathology since dephosphorylation of AD-derived hyperphosphorylated tau strongly reduced propagation after intracerebral injection in mice expressing wildtype tau [73].

Other PTMs include ubiquitination, acetylation, methylation, truncation, sumoylation, nitration, oxidation, glycation, glycosylation, etc. [66].

Altogether, in addition to phosphorylation, some PTMs on specific residues including methylation (Lys163, Lys174, Lys180 and Lys254), acetylation (Lys280) and nitration (Tyr29) might be linked to tau aggregates [47, 78, 109, 130]. Nevertheless, it should be noted that acetylated tau is associated with many tau inclusions in tauopathies [77] but acetylation of tau has been also reported to inhibit tau phosphorylation and its aggregation [30]. This is also true for phosphorylation, when for instance, KXGS sites are phosphorylated [115].

Accumulation of $\mathrm{N}$ - and C-terminally truncated forms of tau has been observed in several tauopathies [141, 150], and some of them can be generated by caspase [49] or calpain proteolysis. In vitro studies indicate that truncated tau might be more prone to aggregation than full-length tau. Native tau filaments extracted from AD brain (bearing all posttranslational modifications) efficiently induce endogenous tau seeding both in cultured cells [45], and after intracerebral injection in tau transgenic mice [79] and in wild-type mice [12]. Interestingly, such native filaments are more efficient at inducing 
seeding than preformed filaments (PFFs) [45, 65], although it is not clear which posttranslational or conformational changes confer this increased efficiency. This might be related to the reported increased resistance to proteinase $\mathrm{K}$ digestion and guanidine hydrochloride solubilisation of recombinant tau aggregates compared to tau aggregates from transgenic mouse brains [45], suggesting that more stable tau aggregates have lower seeding activity.

Altogether, these data indicate that (i) there is hyperphosphorylation in tauopathies and (ii) new phosphorylation sites and/or conformational epitopes, referred to as abnormal phosphorylation and/or conformation, are encountered in tau assemblies in tauopathies. (iii) Other PTMs are also present in these tau assemblies.

\section{Recommendations and cautionary notes:}

1. Reports should be clearer when describing pathological tau in their models so that the detection of certain phosphotau epitopes is not confounded with hyperphosphorylation or aggregation. A more neutral term could be "abnormal phosphorylation" and the term "aggregation" in relationship with phosphorylation should only be used when genuine aggregates have been observed.

2. It is evident from the literature discussed above that interpretation of PTMs assigned to tau in both physiological and pathological conditions is tricky because such changes are crucially dependent on post mortem delay, a factor that is not always taken into consideration. This should be stated and accounted for in future reports. Ideally future studies investigating PTMs of tau would benefit from the inclusion of comparative elements in physiological situations in order to determine whether the modification described is a de novo pathological PTMs or the exacerbation of a physiological PTMs.

3. Analyses of PTMs of tau in murine models are not necessarily transposable to humans since the enzymatic content ensuring these PTMs is different.

Impact of tau mutations and tau levels on tau aggregation MAPT mutations in familial tauopathies have a primary effect at the protein level or affect alternative splicing of tau pre-mRNA. These mutations have various functional effects: they can reduce in vitro the ability of tau to promote microtubule assembly or promote tau assembly into filaments and different types of tau inclusions are found in neurons and glial cells. Many frontotemporal dementia with parkinsonism-17 (FTDP-17) exonic mutations were shown to accelerate tau aggregation in vitro and their overexpression in transgenic animals is currently used to generate models that develop tau aggregates with aging. These models have proven to be very useful for analysis of cell-autonomous pathological cellular mechanisms associated with development of tau aggregates [39]. It seems more difficult to assess the respective roles of cell-autonomous and cell nonautonomous mechanisms in propagation of tau pathology in these mutant tau models compared to propagation in wild-type models $[12,40]$, since in the latter propagation is not dependent on the expression of an aggregationprone mutant tau generating cell aggregates independently of seeding by propagation. Future studies should explore this to understand the differential contributions of cell-autonomous and non-autonomous mechanisms to spread of tau pathology. Some exonic or intronic tau mutations result in imbalance in $4 R / 3 R$ tau ratios (with most often excess of $4 R$ tau) $[74,120]$ without affecting total levels of tau. The in vivo mechanism by which such an imbalance favours tau aggregation is not clearly understood.

Microduplication of $17 \mathrm{q} 21.31$, which encompasses $M A P T$, has been reported to cause an AD-like phenotype with increased tau mRNA [89], but more studies are required to establish the pathogenicity of MAPT duplication. Overexpression of tau in FTDP-17 models is non-physiological and this should be carefully considered when analysing mechanisms of tau aggregation and modulation of tau aggregation. Interestingly, tau aggregation was not observed in a P301L tau knockin mouse expressing tau at physiological levels [51]. Transgenic animal models overexpressing full-length wild-type tau might develop a pathological phenotype but do not however systematically develop tau filaments $[17,62,123]$. Tangle formation from wild-type human tau has been reported in one rodent model in which all six isoforms of wild-type human tau were expressed on a null rodent tau background [8]. This implies that an interplay between the various tau isoforms plays a role in the mechanism by which they aggregate.

Recommendation: The respective roles of presence or absence of tau mutations, levels of expression of tau species, tau isoforms expressed in different animal models need to be carefully interpreted before considering tau aggregation mechanisms in these models validated for human diseases.

\section{Role of other neurodegenerative-related proteins in tau aggregation}

The simultaneous occurrence of several types of protein inclusions made of different aggregate-prone 
proteins characteristic of proteinopathies (alpha-synuclein ( $\alpha$-syn), TAR DNA binding protein-43 (TDP-43), Abeta peptide $(A ß))$ in tauopathies and other neurodegenerative diseases has been observed regularly [121], suggesting that they might mutually interact. E.g. there is experimental evidence that injection of aggregated $A ß$ in mutant tau transgenic mice $[15,63]$ promotes tau pathology at the site of injection, but also in regions remote from the injection site, reminiscent of homotypic tau-seeded tau aggregation. Crossing between mutant amyloid precursor protein (APP) and tau mice $[69,90]$ enhances spreading of tau aggregation [108]. The mechanisms behind this enhancement might reflect cross-seeding phenomena. Cross-seeding of tau with $A ß$ and increased tau spreading after injection of $A ß$-seeded tau have been observed [133]. Similarly cross-seeding between $\alpha$-syn and tau has been demonstrated in cell free assays, and pre-aggregated $\alpha$-syn PFFs induced increased tau aggregation in primary neurons and in mutant transgenic mice in vivo [64]. The relevance of these findings for the in vivo initiation of tau aggregation and enhancement of tau seeding in tauopathies is still poorly understood but might be a critical issue. E.g. in $\mathrm{AD}$, the spatial and chronological progression of tau and amyloid pathologies differs initially $[16,129]$ but their co-existence and development in advanced cases (especially in cortical areas) might reflect a cross-talk.

\section{What is the evidence for prion-like propagation of tau aggregates?}

A key, defining feature of prion-like behaviour is the stable propagation of distinct misfolded protein conformations. To demonstrate that tau aggregates engage in such prion-like behaviour, evidence of cellular uptake, templated seeding and subsequent intercellular transfer of the ensuing newly formed aggregates to induce similar aggregation in recipient cells is required. This section presents the evidence to support this notion from cell and animal models of tauopathy, and discusses whether such prion-like propagation underpins the spread of tau pathology in the brains of tauopathy patients.

\section{Evidence for tau-induced seeding: cellular uptake and induction of aggregation}

Seeding is the induction of aggregation of soluble tau by abnormal tau. The first step in this process is uptake of tau seeds by cells and subsequent templated aggregation and conversion of non-aggregated tau within those cells; i.e. the induced tau aggregates should physically resemble the parent seeds and also be able to induce aggregation of non-aggregated tau. There is evidence that tau seeds fulfil some, but not all of these criteria, as discussed below.

There is little doubt that aggregated tau can be taken up by cells through specific mechanisms (Fig. 2). Uptake of tau aggregates is by macropinocytosis $([45,71]$ and requires heparan sulphate proteoglycans (HSPGs) [71]. Following uptake, tau seeds are in endosomes and must have access to the cytosol to induce aggregation of non-aggregated tau, and the latter mechanisms need to be explored.

Evidence for tau-induced seeding comes from numerous studies conducted in both cell and animal models (e.g. $[25,26,45,76,83,112,142]$. As outlined in Table 2, in these studies, tau seeds were derived from brain homogenates of tauopathy patients or symptomatic tau transgenic mice, cell and conditioned media from tau-aggregate bearing transfected cells, or generated from recombinant tau in vitro. Induction of aggregation was assessed using cellbased fluorescence assays [112, 142], biochemical insolubility assays [45] and immunohistochemical detection of disease-associated pathological tau inclusions $[25,26,76]$. In all instances tau aggregates were seed-competent, though to different degrees. This in itself may shed light on the mechanisms and factors promoting tau-induced aggregation in a pathological context. For example, native pathological tau aggregates derived from brain lysates exhibited 10 fold more seeding ability than equivalent aggregates generated in vitro [45].

Some studies also provide evidence that seeded tau aggregation is templated. Falcon et al. for example showed that native P301S tau seeds derived from transgenic mouse brains conferred their greater seeding competence to the less competent recombinant P301S tau seeds if co-incubated with them in vitro [45]. At the light microscopic level, tau aggregates induced in cells or in vivo have the same morphological appearance as the parent tau seed, and this also hints at a templated mechanism of conversion. This has been shown in many studies from the Diamond lab where morphologically distinct tau accumulates seed formation of accumulates that resemble the parent tau seed both in cell culture [112] and more recently in vivo [83]. That this may be relevant for tauopathies in humans has been demonstrated by the studies from the Goedert and Tolnay labs wherein injection of brain homogenates from different tauopathies into the brains of mice expressing nonaggregated human tau led to formation only of the corresponding tauopathy's inclusions [26].

The evidence described above makes a strong case for tau aggregates engaging in prion-like seeding behaviour, but this is mostly derived from experimental models. To be certain that this is how aggregated tau behaves in human brain, one would need to demonstrate seeding activity in tau aggregates 


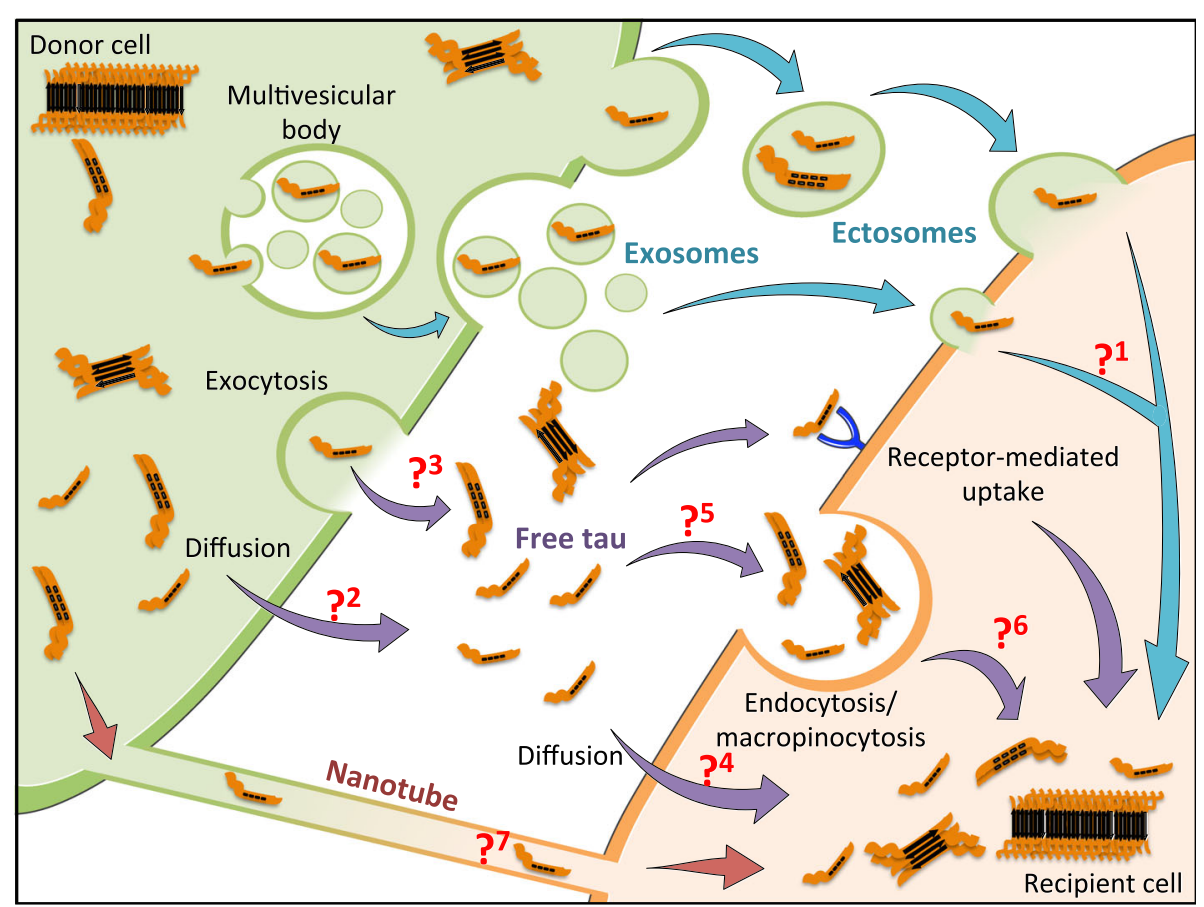

Fig. 2 Transcellular transfer of tau: potential mechanisms underpinning this process. Tau proteins can be transferred from donor cells (green) to recipient cells (orange) using different routes. This figure highlights different pathways reported (blue or violet arrows) or hypothesized (red arrows) in the literature. Whether these pathways are used for physiological transfer of tau proteins to subserve as yet unknown functions of normal tau, or are pathological routes for transfer of tau seeds that can propagate transcellular transfer of tau aggregation, remains to be resolved. Pathway indicated by blue arrows - tau proteins are released in the medium by extracellular vesicles like exosomes and ectosomes. It is unclear how tau proteins carried inside vesicles reach the cytoplasm of recipient cells (Q1). Violet pathway- Around 90\% of tau in the extracellular space is found as free protein. The mechanism(s) by which tau reaches the extracellular space in free form is unknown. Passive diffusion facilitated by a membraneous transporter/receptor $(\mathrm{Q} 2)$ or active exocytosis $(\mathrm{Q} 3)$ might contribute to this process. Uptake of free tau species by recipient cells, including HSPG or APP-mediated endocytosis/ macropinocytosis of tau accumulates have been reported. Whether free or aggregated tau is taken up by other mechanisms such as diffusion (Q4) or non-receptor mediated endocytosis/macropinocytosis (Q5) has not been resolved. Nor is it known how membrane-bound tau can escape from vesicles and enter the cytoplasm of recipient cells (Q6). Orange pathway- Tau was shown to be present inside nanotubes connecting cells in vitro and to allow its interneuronal transfer. This mechanism could potentially participate in prion-like propagation of tau pathology but whether it is a mode of transcellular transfer of seeding-competent tau species in vivo needs to be investigated

derived from tauopathy patients. Support for this has begun to emerge. Sarkosyl-insoluble PHFs extracted from $\mathrm{AD}$ brain tissues induce seeding in cultured cells and in wild-type mice [12, 65]. Moreover, tau seeds capable of inducing aggregation of nonaggregated tau in FRET based biosensor assays have been detected in brain homogenates and cerebrospinal fluid (CSF) of AD cases [48, 84] and in brain homogenates of Pick's disease cases [111]. Thus there is circumstantial evidence that prion-like tau seeds are present in brain tissues in tauopathies and can promote tau aggregation.

\section{Evidence for prion-like spread: Propagation of tau aggregates and their release}

The studies discussed above make the case that tau seeds can induce further aggregation and thus exhibit some "prion-like" property. However, what is the evidence that such tau seeds engage in the other prionlike property, that of propagating their conformation? For this, one needs evidence of transfer of tau aggregates between cells, including secretion and uptake of seeds. Before discussing this evidence, one must explore the potential role, if any, of physiological tau release, since this too will require transcellular movement of tau.

\section{Physiological tau secretion}

There is much evidence that in physiological conditions, tau is secreted and found outside cells in the absence of cell death. This secretion appears to occur independently of tau pathology and may thus highlight a new function for tau. It is regulated by neuronal differentiation and activity [34, 41, 136, 144] and by the small GTPase Rab7A involved in the trafficking of endosomes, autophagosomes, and lysosomes [110]. In addition, MAPT mutations seem to impact tau release in vitro as well as in vivo $[40,82]$, strengthening the concept that wild-type 
Mudher et al. Acta Neuropathologica Communications (2017) 5:99

Page 9 of 20

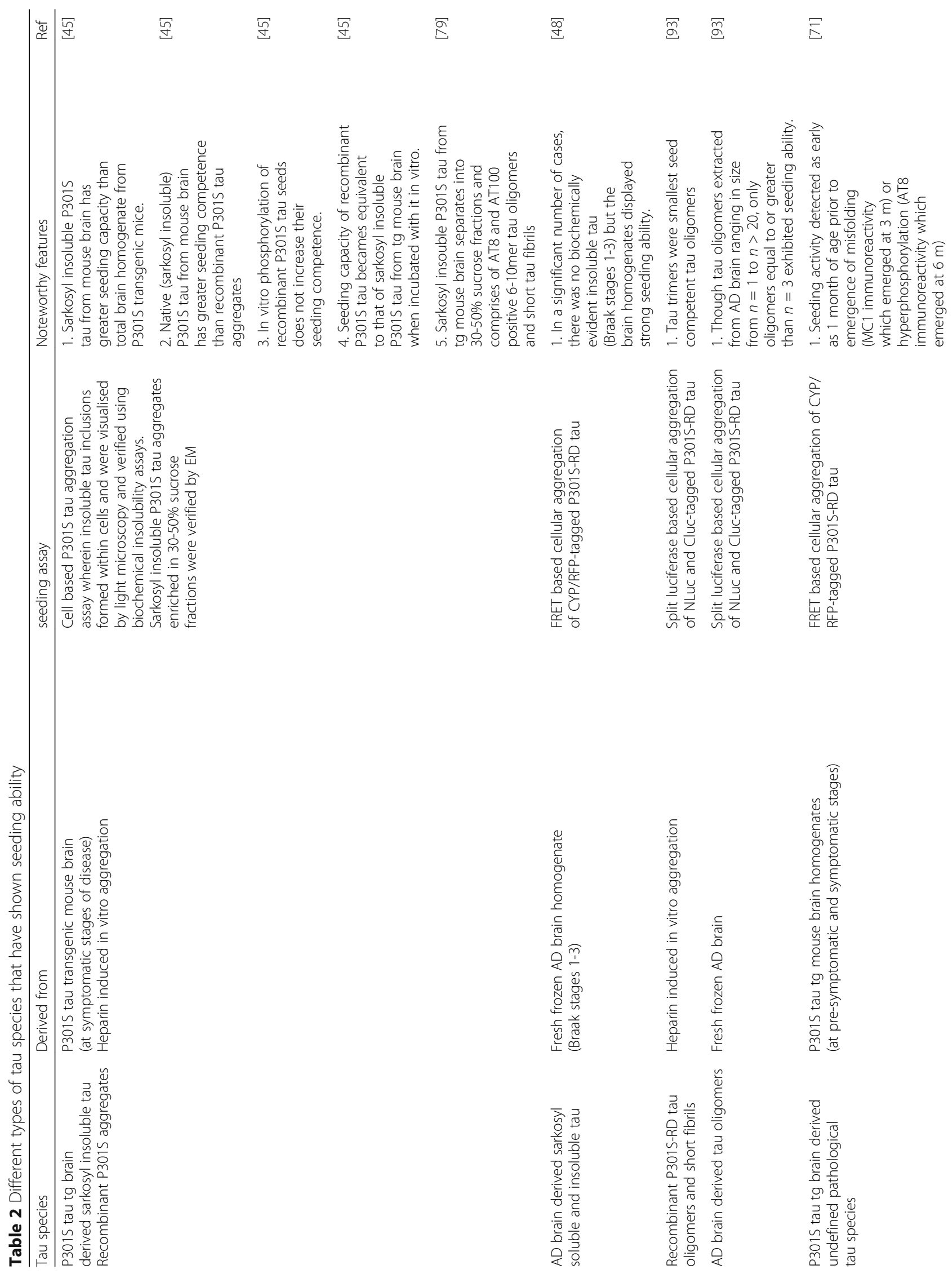


Mudher et al. Acta Neuropathologica Communications (2017) 5:99

Page 10 of 20

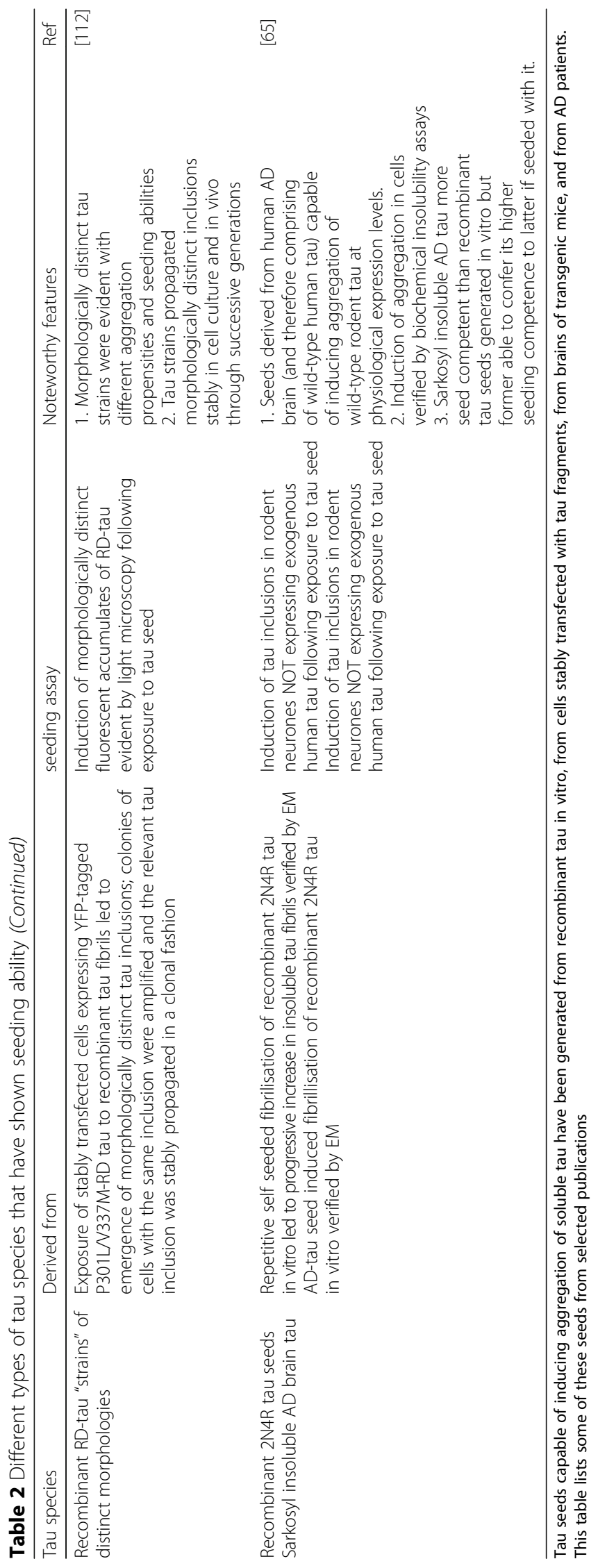


tau associated with sporadic disease and mutant tau linked to FTDP-17 present different characteristics. Mechanisms underpinning tau secretion are still poorly understood but there are a number of cellular pathways implicated. At least $90 \%$ of tau is secreted in a free form $[41,136]$ but release is also mediated by vesicles such as exosomes [11, 119, 136] and ectosomes [41] (Fig. 2).

Tau is mostly secreted in a monomeric and/or truncated non-phosphorylated form [94, 105, 107] raising questions about the role of such secretion in the prionlike propagation of tau pathology. However, recent findings demonstrate that exosomes derived from $\mathrm{N} 2 \mathrm{~A}$ neuroblastoma cells overexpressing repeat domain deltaK280 fragments, contain tau aggregates and that these exosomes mediate tau aggregation in receiving cells [136]. In the same way, exosomes containing tau with seeding activity have been isolated from brains of tau transgenic mice, but not from biological fluids [106]. Tau in both free and vesicular forms has however been detected in CSF and interstitial spinal fluid (ISF) from both animal models $[41,143]$ and patients $[48,136]$ and in the latter this tau was seed competent.

These studies imply that tau in extracellular vesicles can act as a seed and therefore contribute to prion-like propagation of tau pathology but this has to be demonstrated in vivo. Additionally, whether secreted free tau can also act as a seed, has yet to be determined.

It is entirely conceivable that tau released in physiological conditions is not abnormally folded or otherwise pathological, and therefore cannot act as a seed for the propagation of tau pathology. Perhaps under pathological conditions, aggregated tau is released, which is capable of driving the transcellular propagation of pathology.

\section{Recommendation}

Future studies should aim to decipher the mechanism(s) regulating tau secretion in physiological conditions, whether they are conventional or unconventional, and what role if any, they play in transcellular propagation of tau pathology in disease. In a pathological context, extracellular tau might also be related to clearance independently of any propagation/transfer to neighbouring/ connecting cells. It is vital to take these parameters into account before designing therapeutic interventions targeting extracellular tau.

\section{Transcellular tau transfer}

As discussed above, multiple studies in cell culture demonstrate that tau seeds can be released from donor cells and taken up by recipient cells where aggregation of nonaggregated tau is induced. Transcellular transfer of tau aggregates has been demonstrated between cells cultured serially in microfluidic chambers [142]. The spread of tau from neuron to neuron across trans-synaptic connections via exosomes, capable of seeding aggregation has been reported $[106,136]$. Other mechanisms do not require secretion but a direct connection between cytoplasms. These structures, called nanotubes have been described in various cell types, including neuronal and immune cells. Recent papers showed that nanotubes support interneuronal transfer of tau fibrils in neurons $[1,128]$. Whether such structures exist in the brain remains to be elucidated and whether the transfer of such seeds mediates aggregation in the recipient cells is still not demonstrated. These studies illustrate that there are many ways for tau to be secreted but it is not clear how efficient each of these is in terms of transfer of tau seeds. For instance though $90 \%$ of tau is secreted in a free form with only a minority being secreted from ectosomes, the latter are more efficient for transcellular tau transfer. This highlights the importance of understanding which mechanism(s) of trans-cellular tau transfer plays a role in physiology and which may become more prominent in pathology.

Only a few papers address the actual mechanism(s) regulating cellular tau uptake. Free tau has been shown to bind receptors on receiving cells, including HSPGs [71] and APP [125], which are proposed to enable cellular entry via receptor-mediated endocytosis. To date, nobody has investigated the mechanism(s) regulating vesicular tau uptake. Nevertheless, tau seems to be able to activate signal transduction pathways in receiving cells leading to $\mathrm{Ca}^{2+}$ release and neuronal damage $[59,60]$. The latter studies highlight pathogenic effects of extracellular tau other than those required for prion-like behavior. Whether or not such effects of extracellular tau are involved in spread of tau pathology remains to be determined.

\section{Neuroanatomical spreading of tau aggregates}

The cellular studies described above make a case for transcellular transfer of tau pathology. Supporting these findings, trans-synaptic propagation of tau pathology has been demonstrated using a variety of different approaches in transgenic mice. Pioneering research in this field, Clavaguera et al. showed not only the induction of tau aggregation in rodent brain following intracerebral injection of brain homogenates containing tau seeds, but also the time-dependent appearance of tau pathology in anatomically connected brain regions [25]. Others have also reported the appearance of tau pathology in areas connected to the sites injected with tau seeds or viral vectors expressing tau $[3,40,76,102]$. De Calignon et al. [36] and Liu et al. [91] used a model in which expression of human tau was restricted to the entorhinal cortex only and yet tau pathology was evident in anatomically linked regions not expressing the human tau trangene. 
This could only have happened if tau seeds generated in the entorhinal cortex had propagated to induce aggregation in brain regions connected to the entorhinal cortex. Studies in tau transgenic mice indicate that tau seeds predict spread of disease by appearing in brain regions prior to appearance of any other pathological change [72]. Moreover, another study in a rodent model showed that if one of the hypothesised modes of transsynaptic transfer of seeds, exosome synthesis is inhibited, propagation of tau pathology is significantly suppressed [11]. Propagation of abnormal tau in a P301L model without expression of endogenous tau has been observed, suggesting that spreading of tau does not always require templated misfolding of endogenous tau [137].

Thus there is persuasive evidence to support the concept that seed competent tau aggregates can be taken up by cells, induce aggregation of non-aggregated tau and then get released by cells. This process might be repeated across synaptically linked networks and in this way tau pathology spreads through neuroanatomically linked brain regions. However, for this to be relevant to the mechanism by which tau pathology spreads in humans, one would need evidence that this occurs in the brains of patients. The fact that tau pathology spreads in a defined neuroanatomically connected pattern in $\mathrm{AD}$, as described by the Braak staging is tentative evidence for trans-synaptic spread of pathology. However, the sequential appearance of tau pathology in anatomically connected regions may simply reflect a spatiotemporal vulnerability of different anatomical regions to tau aggregation, unrelated to propagation. A recent study reporting seeding behaviour in brains of AD patients argues against this [48]. In this report, seeding ability in different brain regions correlated positively with Braak stage, negatively with MMSE scores, and preceded overt tau pathology. This strongly implies that spread of tau pathology occurs by a prion-like transsynaptic mechanism. It also explains the histopathological observation made by Duyckaerts et al. 20 years ago when the concept of prion-like propagation of non-prion protein aggregates was in infancy [42]. They reported the conspicuous absence of tau pathology in a frontal cortical region that had been anatomically disconnected from the limbic region as a result of neurosurgery decades before the patient developed AD. This was despite extensive pathology in immediately adjacent brain regions as well as in the limbic and isocorttical areas. Together these two studies in AD patients provide the most compelling evidence that prion-like propagation of tau pathology occurs in human brain and underpins the characteristic progression of pathology in tauopathies.

Thus collectively, there is ample, albeit circumstantial evidence to support the case that prion-like propagation contributes to the spread of tau pathology in tauopathies.

\section{Cautionary notes and recommendations}

1. It must be noted here that prion-like behaviour should not be extended to the infectivity behaviour of prions. Prions induce templated misfolding of a normal prion protein, the propagation of this misfolding in the brain, across tissues (e.g. from periphery to brain) and between organisms. Prion-like behaviour of tau for now is mainly documented by templated seeding and propagation of aggregation across brain areas. Some animal experiments suggest however that an intracerebral tauopathy can develop after peripheral administration of tau aggregates [27]. Careful analysis of the different steps of these complex processes is needed for all molecules exhibiting prion-like behaviour and for differentiating them form infectious entities [43].

2. Some of the animal models that have thus far been used to demonstrate transcellular spread of tau seeds have limitations, which may confound the evidence that they provide and this should be considered. For example:

a) Many models use intracereberal injections of tau seeds in the form of different brain fractions or recombinant tau. This material will diffuse some distance away from the injection site. Although this might result in uptake of tau seeds and intracellular seeding, it does not necessarily imply transcellular spread and propagation through neuroanatomically connected areas of aggregated tau. This should be taken into consideration when interpreting their data.

b) The animal models that utilise tissue-specific transgene expression use drivers that have been reported to be leaky [146]. Even if this occurs to a very small extent, it gives rise to the possibility that some of the induction of aggregation evident in distal regions arose due to small amounts of transgene expression there rather than seed propagation from the tissue of greatest transgene expression.

\section{Evidence for trans-synaptic and non-synaptic transmission of tau pathology}

The studies described above make a strong case for the propagation of tau pathology to be mediated by synaptic mechanisms. However even in the prion field, where this concept emerged, there are indications that transsynaptic propagation may not be the only means of spread of prions [116]. In the tau field too, it may be imperative to keep an open mind in this regard and entertain the idea that the spread of tau pathology through the brains of tauopathy patients may occur by a number of means including trans-synaptic spread, interstitial 
diffusion and even microglial intervention. To this end, one study has shown that microglial depletion or suppression of production by microglial cells of exosomes significantly reduces the propagation of AT8-positive oligomers in a rodent model [11]. There is precedence for glial cells playing a role in transcellular transmission of Huntingtin aggregates in a Drosophila model [101]. The demonstration of a similar contribution of glia in propagation of pathological tau suggests that glial cells may play a significant role in transcellular transfer of pathological aggregates in many proteinopathies.

Propagation through both anterograde and retrograde pathways between connected cells might occur, potentially through different mechanisms, and this needs further investigation.

\section{The nature of the tau species that spread/ propagate}

Tau seeds derived from multiple sources have been used and in all cases shown to be competent at inducing tau aggregation and transcellular propagation of aggregation. This includes seeds generated by incubating recombinant tau protein with fibrillization inducers in vitro, or from transfected neuronal and non-neuronal cell lysates and conditioned media, or obtained from brain homogenates of transgenic mice and human tauopathy brains. Tau seeds have also been generated in vivo following the injection of viral vectors expressing tau into rodent brain. Though the nature of the tau species may differ between these studies, and though there may be multiple tau species with seeding ability in human brain, one can infer the properties all seed-competent tau species have in common.

Various studies have shown, perhaps unsurprisingly, that monomeric soluble tau or mutant tau incapable of aggregation has no seeding ability $[45,93]$. There are however conflicting reports as to the minimal number of tau units required to confer efficient seeding ability. Recent findings showed that monomeric tau (exhibiting unique structure with accessible VQIINK and VQIVYK motifs) derived by sonication from fibrillized tau, seeded aggregation of tau in cells (http://www.alzforum.org/ news/conference-coverage/monomeric-seeds-and-oligomeric-clouds-proteopathy-news-aaic). One study reported that a minimum of 3 tau molecules is required [93], whilst another report only showed seeding when 6-10 tau molecules were aggregated [79]. Nonetheless both studies showed that larger oligomers comprising between 10 and 100 tau units display greater seeding competence [93]. In line with this study, others too have shown that tau oligomers can induce seeding, both in cell culture [136] and in vivo [22].

As well as for oligomers, seeding capability of larger insoluble tau aggregates has also been characterised. Jackson et al. [79] characterized the seed-competent native tau species from the brains of P301S tau mice, and showed that a range of short tau fibrils, including short filaments and ring-like structures, phosphorylated at the AT8 and AT100 sites, were endowed with the greatest seeding capacity [79].

The studies above have extensively characterised tau seeds generated often in experimental models. However, for this to be relevant to humans, future studies would need to compare and contrast these experimental tau seeds with those found in the brains of patients with different tauopathies. This has already begun, and early indications are that tau seeds found in $\mathrm{AD}$ brain can be isolated from both soluble and insoluble brain fractions [48]. This implies that there are multiple tau seeds in existence in diseased human brains that comprise both soluble and insoluble tau aggregates.

Summarising these studies, one can therefore conclude that monomeric/oligomeric/ multimeric tau species with a unique conformation might be the only requirement for initial tau seeding behaviour. Beyond this, other characteristics of the tau seed, such as how large it is, PTMs and how it is generated i.e. whether it is natively formed or generated in vitro, will determine seeding competence. Ascertaining which tau species are the key-"building blocks" of filaments is an issue. The nature of the conformation will also play a part with the general assumption that beta-pleated sheet content is a determinant for seeding capability. It will be important to identify and characterise the various tau seeds found in tauopathies and the factors that regulate seeding behaviour, if this is to be an avenue for preventing propagation of tau pathology in tauopathies.

\section{What is the evidence for "tau strains or conformers"?}

Prion strains with distinct biological properties can explain the variations in clinical presentations and in neuropathological lesions in prion diseases [29]. Similarly, the notion that tau "strains" exist might explain peculiar patterns of neuropathological lesions in different tauopathies, and the intensity and the kinetics of disease progression [28]. A given strain may determine where the disease process starts, followed by prion-like spreading based on the connectivity of the primarly affected regions. Experimental injections of various types of brain extracts from patients with different tauopathies in animal models induce different patterns of tau pathology, cellular and neuropathological lesions $[26,83,84]$. The treatment of cultured cells with similar extracts induces the formation of different types of inclusions [112] and cell based assays to measure tau seeding activity will be helpful to characterize tau strains [84].

These observations are compatible with the existence of different tau strains. As for prions, different structural 
and biochemical features might define these strains. Distinct conformations evidenced by a relative resistance to proteolytic enzymes are such features. The definition of a molecular conformer might fundamentally be structural and future cryo-EM studies may well provide this information. There are likely to be different molecular conformers made of $3 R+4 R, 3 R$ and $4 R$ tau isoforms. Protein sequence and mass spectrometric analyses have revealed that the protease-resistant core units of tau aggregates differ between tauopathies [127]. It also remains to be seen if different strains of aggregated tau exist within these categories, for instance between $\mathrm{AD}$ and chronic traumatic encephalopathy, or between PSP and CBD.

Many of the present studies were done at the light microscopy level and more work is needed to document the structural characteristics of tau strains in different tauopathies. Tau assemblies with distinct conformations that do not stably propagate their properties in vivo are probably not bona fide strains. Like for prions, it is expected that a defined tau strain will recapitulate similar patterns of neuropathological lesions and keep its biological properties after serial passage in animal models. Whilst this has been shown for some tau accumulates $[83,84,112]$, it is not yet clear that it is a property shared by all seed-competent tau conformers.

\section{What is the evidence that propagation of tau aggregates is toxic?}

Dissociation between aggregation, propagation and toxicity

The general assumption is that propagation of tau aggregates is synonymous with the propagation of toxicity. This is because tau aggregates are believed to be toxic, so one would assume that their induction and propagation would cause dysfunction and degeneration of the neuronal networks through which they spread.
Supporting this, some studies have shown that seeding ability of different recombinant tau seeds correlates with their toxicity in vitro [112] and to some extent in vivo [83]. Another study has shown both electrophysiological deficits and resultant behavioural dysfunction following induction of templated tau seeding [124]. However, this has not been assessed in the majority of studies showing prion-like propagation of tau pathology in vivo. Degeneration of tangle-bearing neurons has been described when the tau seeds induced tangle-like pathology in neurons in the locus coeruleus after injection in this area [76]. Others clearly state that there was no degeneration despite clear propagation of tau aggregates $[3,137]$. This is an area that requires further investigation because the relationship between propagation of tau aggregates and tau-induced degeneration is not clear. In the prion field too, the distribution of misfolded prion protein $\mathrm{PrP}^{\mathrm{SC}}$ alone does not predict neurodegeneration [4].

Toxcity of tau independent of its aggregation is another caveat that should be considered. Tau interactions with other cell components can be toxic to some cellular process, and lead to the spreading of toxicity through signalling mechanisms.

\section{Different pathological tau species employ different mechanisms of toxicity}

Perhaps a lack of clarity arises because different pathological tau species might use different mechanisms of toxicity (Table 3). Soluble hyperphosphorylated tau species (which may be monomeric or small oligomeric aggregates) cause neuronal dysfunction characterised by breakdown of cytoskeletal integrity, disrupted axonal transport [100] and synaptic dysfunction in Drosophila [32, 97]. This is perhaps more accurately described as "phospho-tau mediated dysfunction" rather

Table 3 Potential modes of tau toxicity

\begin{tabular}{|c|c|c|}
\hline Pathological change and Tau species implicated & Potential modes of tau toxicity & $\begin{array}{l}\text { Selected } \\
\text { References }\end{array}$ \\
\hline $\begin{array}{l}\text { Hyperphosphorylation (e.g. soluble } \\
\text { monomer/dimer) }\end{array}$ & $\begin{array}{l}\text { Loss Of microtubule-binding (and other) Function(s) (LOF) } \\
\text { leading to axonal transport and synaptic defects reflected } \\
\text { in mitochondrial clumping, Golgi disruptions and mis-sorting } \\
\text { of synaptic proteins. Mis-localisation may also be evident causing } \\
\text { Gain Of toxic Function (GOF). Collectively these may be responsible } \\
\text { for neuronal dysfunction at early stages of disease. It is possible } \\
\text { that a partial LOF is required for, and leads to an eventual GOF }\end{array}$ & {$[31,32,52,97,100]$} \\
\hline $\begin{array}{l}\text { Misfolding/aberrant folding and aggregation into } \\
\text { small aggregates (e.g. sarkosyl soluble oligomers) }\end{array}$ & $\begin{array}{l}\text { Neuronal dysfunction and neurodegeneration evident in } \\
\text { some models in the absence of larger aggregates implying that } \\
\text { smaller soluble oligomeric species responsable for these phenotypes }\end{array}$ & $([53,87])$ \\
\hline $\begin{array}{l}\text { Aggregation (into large insoluble oligomers such } \\
\text { as granular tau oligomers and filaments including tangles) }\end{array}$ & $\begin{array}{l}\text { Space-occupying lesions resulting in GOF. Toxicity debated because } \\
\text { in some models rescue of neuronal dysfunction and degeneration } \\
\text { evident despite persistence of larger aggregates. }\end{array}$ & $\begin{array}{l}([14,31,33,52 \\
114,122,140])\end{array}$ \\
\hline
\end{tabular}

The various pathologial changes in tau may be responsible for causing loss of normal function (LOF) or gain of toxic function (GOF). In the face of emerging novel functions of tau, there may be numerous modes of toxicity via a number of LOF mechanisms. Toxicity resulting from GOF mechanisms are more difficult to dissect but based on reports of neuronal dysfunction or neurodegeneration in the absence of large insoluble tau filaments, the tau species responsible (or not as the case may be) are begining to be understood. Recommendation: Future studies should seek to clarify terminology and consistency in ascribing modes of toxicity to tau species 
than overt toxicity. Tau oligomers and small tau fibrils, which are most likely to engage in transcellular propagation, are associated with toxicity arising from gain of toxic function mechanisms [53, 87]. Whether this is due to the transcellular propagation of aggregation is not proven and further work is required to understand the connection between these two phenomena. In contrast, the toxicity of larger insoluble oligomers and tangle-like structures is debatable with some studies stating that they are toxic whilst others implying that they are protective $[14,31$, $33,52,114,122,140]$. Clearly different pathological tau species are differentially toxic and which species forms at which time point in the neuronal circuit through which the tau pathology is spreading will determine tau toxicity. Moreover, within these neurons, different forms of tau could be responsible for templated propagation of pathology and tau-induced neuronal dysfunction.

\section{Recommendation}

Terminology used to describe tau toxicity should be clarified and consistency sought. This will enable a fuller picture to be built to enable understanding of which pathological tau species (hyperphosphorylated monomer, oligomer, fibril) is responsible for which effect (dysfunction, degeneration, propagation).

\section{What is the role of genetic factors in the propagation of tau pathology?}

Genetic and genome-wide association (GWAS) studies have identified risk factors for AD [132] and other tauopathies [70]. These genes are implicated in many different cellular processes but how they affect these processes is not known. Genes products involved in exocytosis and endocytosis, the function of synaptic vesicles, protein clearance, intracellular transport, etc. might be directly linked to the propagation of tau pathology [13]. Much experimental work is still needed to link the effects of these gene variants to increased or decreased propagation of tau pathology.

Some GWAS have identified direct (or indirect) binding partners of tau (APOE, BIN1, PICALM, CLU). Inheritance of the APOEe4 is a well-established risk factor for $\mathrm{AD}$ and tau-mediated neurodegeneration is aggravated by APOE4 [118]. A reduction in insulin signaling in the brain is a feature of AD and APOE4 impairs neuronal insulin signaling [148]. BIN1 is a negative regulator of clathrin-mediated endocytosis and modulates tau toxicity [23]. The level of neuronal-specific BIN1 isoform is reduced in $\mathrm{AD}$ brain and lower levels of BIN1 promote propagation of tau pathology in cultured neurons [21]. PICALM, which is also involved in clathrin-mediated endocytosis, modulates autophagy and alters the clearance of tau [95]. PICALM levels are decreased and
PICALM co-localizes with tau inclusions in AD [6] and in other tauopathies [7]. PTK2B, involved in a cell adhesion pathway, was also recently identified as a modulator of tau pathology [38].

MAPT mutations in familial tauopathies favour tau aggregation and determine which type of tau isoforms are included in tau aggregates (see also above paragraph on tau aggregation) [50]. Although this might facilitates propagation of tau pathology, distinguishing the role of cell autonomous mechanisms (e.g. "spontaneous" formation of tau aggregates in different cells independently of their connections) from cell non-autonomous mechanisms (e.g. dependent of transfer of pathological tau between connected cells) in tau spreading might be difficult.

\section{Future directions}

How can future studies enhance our understanding of propagation of tau pathology?

Need to better understand the role of prion-like propagation of tau pathology in human tauopathies

A fundamental question is whether prion-like mechanisms play a role in sporadic human tauopathies and other neurodegenerative diseases. Although prion-like mechanisms may also operate in familial forms of these diseases, they may not be required there, because all cells express the mutations. Further studies are needed to clarify if some cases of sporadic tauopathies can be acquired though it would require unusual conditions. A difference between sporadic tauopathies and prion diseases is the lack of evidence for acquired cases in the former. However, recent findings may begin to provide this evidence. Recent work, in the UK, on people who had received cadaver-derived human growth hormone as children, which was contaminated with prions (and possibly $A D A ß$ seeds), developed a prion disease and showed some $A \beta$ deposits at autopsy. However, they had no tau inclusions, nor did they suffer from $\mathrm{AD}$ [80]. In another series of French patients who died from iatrogenic Creutzfeldt-Jakob disease after the injection of cadaver-derived human growth hormone, some cases showed tau inclusions and tau and $A ß$ contaminants were detected in batches of growth hormone (Duyckaerts et al., in press Acta Neuropathologica 2017).

The spreading of tau pathology in the brain during progression of tauopathies is also compatible with selective vulnerability of neuronal populations to pathological processes [135]. This could be true even in the framework of a prion-like mechanism. Connected cells might be differentially sensitive to prion-like mechanisms of release and uptake of aggregates, and to seeded aggregation. Independently or not from prion-like mechanisms, some cells could successfully degrade aggregates for a longer time before becoming affected. This may explain 
the frequently observed age dependence of these human diseases, reflecting the ability of cells to degrade aggregates and prevent spread. These proteins may have an intrinsic tendency to aggregate (although tau is very soluble) and only when the balance between degradation and aggregation favours the latter, will the ordered assemblies of tau be able to spread.

Thus, the respective roles of prion-like mechanisms and selective vulnerability in the development of tau pathology remain to be clarified.

\section{Learn from the prion field}

In the prion field different strains of $\operatorname{PrP}^{\mathrm{SC}}$ are associated with different prion diseases. The "prion-like" behaviour of tau seeds has led to the concept that distinct tau strains underpin different tauopathies. The popularity of this idea is growing fast, and there is a large body of evidence to support many, though not all, of its aspects [135]. However, unlike the prion field where distinct strains of $\mathrm{PrP}^{\mathrm{SC}}$ are evident in different prion diseases, and where degeneration is believed to require spread of $\operatorname{PrP}^{\mathrm{SC}}$, this has to be more clearly demonstrated in tauopathies. Future studies need to identify distinct tau strains in different tauopathies and demonstrate that the spread of tau seeds is required for the propagation of pathology. Added to this, future studies need to focus on understanding the impact of seeding and propagating tau aggregation within affected neural networks to appreciate if this has any clinical significance. A large body of research in the prion field now suggests that the $\operatorname{Pr}^{\mathrm{SC}}$ species responsible for transcellular propagation and neurotoxicity are partially distinct. Since multiple pathological tau species have been described, it is possible that the same is true for tauopathies. This needs further investigation.

Perhaps learning from the prion field will facilitate the studies proposed above. Even though tau seeds may be better described as "propagons" rather than bona-fide "prions", the methods used to characterise and study $\mathrm{PrP}^{\mathrm{SC}}$ will be invaluable for deciphering the "prion-like" properties of tau seeds. Such studies should enable better understanding of the templated seeding behaviour of tau strains, the mechanisms underpinning their internalisation, secretion and eventual transcellular spread, their mechanisms of toxicity and how tightly this is linked to their propagation, and the role of glial cells in their spread. It would also be important to determine the level of amplification of tau seeds during spread, which to date has not been addressed, and which can be assessed using experimental approaches routinely used in the prion field.

\section{Understand physiological role of tau secretion and its relevance to pathological tau spread}

As discussed here, tau can be found extracellularly but it is not clear if this is pathological or indicates an as yet unknown function of tau. Since the two key tenets of the prion-like spread of tau pathology are secretion and uptake of tau species, it becomes imperative to investigate how secretion and uptake of pathological tau seeds differ from secretion and uptake of normal tau. It can be hypothesized that if the tau species released are seed-competent, then this process could contribute to transcellular propagation of tau pathology. However future studies need to demonstrate this.

\section{Developing better models to study tau aggregation and propagation}

Models expressing human wild-type and FTDP-17 tau at normal physiological levels, tau knock-in models [52], models expressing the whole set of human tau isoforms [8] and models reproducing the exact alternative splicing of the human tau gene are promising approaches for the future (http://www.alzforum.org/news/conference-coverage/ next-generation-mouse-models-tau-knock-ins-and-humanchimeras). Grafting human neurons derived from stem cells within animal models, simulating exposure to a complex $3 \mathrm{D}$ in vivo environment is another approach [44]. These second-generation models are expected to provide further insights into the physiopathology of tau aggregation and tau spreading.

\section{Propagation-centred therapies}

The fact that $\mathrm{AD}$ brains and brain homogenates from tauopathy models contain tau seeds capable of inducing tau aggregation in vitro and this correlates with progression of disease state [72, 126], implies that anti-seeding approaches may have therapeutic value. Blocking of tau aggregation and inter-neuronal tau propagation in vitro by anti-tau antibodies has been reported [99]. Anti-tau antibodies capable of suppressing tau seeding activity in vitro effectively reduced tau aggregation in a transgenic model of tauopathy; however whether this was through reduction of propagation needs to be confirmed [145]. Spreading of tau pathology after injection of PFFs in mutant tau mice was significantly reduced by passive immunisation with phosphotau antibodies [113]. The most convincing evidence for a causal role of prion-like spread of tau aggregation in pathogenesis of disease will come if interference with propagation of tau pathology were to suppress neurodegeneration and lead to clinical benefits. Promising results are emerging from mouse models and they may soon translate to patients.

\section{Concluding remarks}

The concept that brain spreading of tau pathology in tauopathy occurs by a "prion-like" mechanism is fast gaining popularity. The key tenets of this hypothesis are that diverse conformational tau strains exist in different tauopathies, and that they drive templated aggregation 
followed by the intercellular propagation of tau pathology. While the evidence in favour of this hypothesis is growing daily, its clinical relevance is still debated [135]. Furthermore, it is not clear how spread of tau pathology through this "prion-like propagation" relates to spread of pathology arising from differential spatio-temporal vulnerability of connected neuronal populations. Future studies which take into account some of the points raised here are needed to document the respective roles of these pathological mechanisms in tauopathies. Nonetheless, since clinical symptoms are likely to manifest only after extensive spread of pathology, understanding all potential modes employable by pathological tau to traverse neural circuits opens novel therapeutic avenues to arrest the spread of tau aggregates at a preclinical stage.

\section{Acknowledgements}

The authors would like to thank Katrin Deinhardt (University of Southampton) for proofreading the manuscript.

\section{Authors' contributions}

$A M$ and JPB devised overall format of manuscript and together with MG, MC and LB wrote the manuscript. All remaining authors contributed to discussion and proof-reading. MG, SD, MC and LB generated figures; AM, JPB and MG generated tables. All authors read and approved the final manuscript.

\section{Competing interests}

All authors declared that they have no competing interests.

\section{Publisher's Note}

Springer Nature remains neutral with regard to jurisdictional claims in published maps and institutional affiliations.

\begin{abstract}
Author details
${ }^{1}$ University of Southampton, Biological Sciences, Faculty of Natural and Environmental Sciences, SO17 1BJ Southampton, UK. ${ }^{2}$ Univ. Lille, Inserm, CHU-Lille, UMR-S 1172, LabEx DISTALZ, 59000 Lille, France. ${ }^{3}$ Department of Neurology, MassGeneral Institute for Neurodegenerative Disease, Massachusetts General Hospital, Harvard Medical School, Charlestown, Massachusetts, USA. ${ }^{4}$ Network Center for Biomedical Research in Neurodegenerative Diseases (CIBERNED), Madrid, Spain; CIEN Foundation, Queen Sofia Foundation Alzheimer Center, Madrid, Spain. ${ }^{5}$ Dementia Research Group, BioMedical Research Institute, Hasselt University, 3500 Hasselt, Belgium. ${ }^{6}$ Institut de Biologie Paris Seine-Laboratoire Neuroscience Paris Seine INSERM UMRS 1130, CNRS UMR 8246, UPMC UM 118 Université Pierre et Marie Curie, Paris, France. ${ }^{7}$ DZNE (German Ctr. Neurodegen. Diseases), Bonn, Germany. ${ }^{8}$ CAESAR Research Center, Bonn, Germany. ${ }^{9}$ DESY, Hamburg, Germany. ${ }^{10}$ MRC Laboratory of Molecular Biology, Francis Crick Avenue, CB2 OQH Cambridge, UK. ${ }^{11}$ Laboratory of Histology, Neuroanatomy and Neuropathology Université Libre de Bruxelles, Faculty of Medicine, ULB Neuroscience Institute (UNI) 808, route de Lennik 1070, Brussels, Belgium.
\end{abstract}

Received: 5 September 2017 Accepted: 30 October 2017

Published online: 19 December 2017

\section{References}

1. Abounit S et al (2016) Tunneling nanotubes: a possible highway in the spreading of tau and other prion-like proteins in neurodegenerative diseases. Prion 10(5):344-351

2. Adams SJ et al (2010) Three repeat isoforms of tau inhibit assembly of four repeat tau filaments. PLoS One 5(5):e10810

3. Ahmed $Z$ et al (2014) A novel in vivo model of tau propagation with rapid and progressive neurofibrillary tangle pathology: the pattern of spread is determined by connectivity, not proximity. Acta Neuropathol 127(5):667-683
4. Alibhai J et al (2016) Distribution of misfolded prion protein seeding activity alone does not predict regions of Neurodegeneration. PLoS Biol 14(11): e1002579

5. Ambadipudi S et al (2017) Liquid-liquid phase separation of the microtubule-binding repeats of the Alzheimer-related protein tau. Nat Commun 8(1):275

6. Ando K et al (2013) Clathrin adaptor CALM/PICALM is associated with neurofibrillary tangles and is cleaved in Alzheimer's brains. Acta Neuropathol 125(6):861-878

7. Ando K et al (2016) Level of PICALM, a key component of clathrin-mediated endocytosis, is correlated with levels of phosphotau and autophagy-related proteins and is associated with tau inclusions in AD, PSP and Pick's disease. Neurobiol Dis 94:32-43

8. Andorfer $\mathrm{C}$ et al (2003) Hyperphosphorylation and aggregation of tau in mice expressing normal human tau isoforms. J Neurochem 86(3):582-590

9. Arendt T, Stieler JT, Holzer M (2016) Tau and tauopathies. Brain Res Bull 126(Pt 3):238-292

10. Arendt T et al (2003) Reversible paired helical filament-like phosphorylation of tau is an adaptive process associated with neuronal plasticity in hibernating animals. J Neurosci 23(18):6972-6981

11. Asai $\mathrm{H}$ et al (2015) Depletion of microglia and inhibition of exosome synthesis halt tau propagation. Nat Neurosci 18(11):1584-1593

12. Audouard E et al (2016) High-molecular-weight paired helical filaments from Alzheimer brain induce seeding of wild-type mouse tau into an argyrophillic 4R tau pathology in vivo. Am J Pathol 186(10):2709-2722

13. Avila J, Gomez-Ramos A, Bolos M (2015) AD genetic risk factors and tau spreading. Front Aging Neurosci 7:99

14. Berger $Z$ et al (2007) Accumulation of pathological tau species and memory loss in a conditional model of tauopathy. J Neurosci 27(14): 3650-3662

15. Bolmont $T$ et al (2007) Induction of tau pathology by intracerebral infusion of amyloid-beta -containing brain extract and by amyloid-beta deposition in APP x tau transgenic mice. Am J Pathol 171(6):2012-2020

16. Braak $\mathrm{H}$ et al (1986) Occurrence of neuropil threads in the senile human brain and in Alzheimer's disease: a third location of paired helical filaments outside of neurofibrillary tangles and neuritic plaques. Neurosci Lett 65(3):351-355

17. Brion JP, Tremp G, Octave JN (1999) Transgenic expression of the shortest human tau affects its compartmentalization and its phosphorylation as in the pretangle stage of Alzheimer's disease. Am J Pathol 154(1):255-270

18. Brion JP et al (1985) Mise en évidence immunologique de la protéine tau au niveau des lésions de dégénérescence neurofibrillaire de la maladie d'Alzheimer. ArchBiol(Brux) 95:229-235

19. Buée $L$ et al (2000) Tau protein isoforms, phosphorylation and role in neurodegenerative disorders. Brain Res Brain Res Rev 33(1):95-130

20. Bussière T et al (1999) Phosphorylated serine422 on tau proteins is a pathological epitope found in several diseases with neurofibrillary degeneration. Acta Neuropathol 97(3):221-230

21. Calafate $S$ et al (2015) Synaptic contacts enhance cell-to-cell tau pathology propagation. Cell Rep 11(8):1176-1183

22. Castillo-Carranza DL et al (2014) Specific targeting of tau oligomers in Htau mice prevents cognitive impairment and tau toxicity following injection with brain-derived tau oligomeric seeds. J Alzheimers Dis 40(Suppl 1):S97-S111

23. Chapuis J et al (2013) Increased expression of BIN1 mediates Alzheimer genetic risk by modulating tau pathology. Mol Psychiatry 18(11):1225-1234

24. Chirita CN et al (2005) Triggers of full-length tau aggregation: a role for partially folded intermediates. Biochemistry 44(15):5862-5872

25. Clavaguera F et al (2009) Transmission and spreading of tauopathy in transgenic mouse brain. Nat Cell Biol 11(7):909-913

26. Clavaguera F et al (2013) Brain homogenates from human tauopathies induce tau inclusions in mouse brain. Proc Natl Acad Sci U S A 110(23): 9535-9540

27. Clavaguera F et al (2014) Peripheral administration of tau aggregates triggers intracerebral tauopathy in transgenic mice. Acta Neuropathol 127(2):299-301

28. Clavaguera F et al (2015) Invited review: Prion-like transmission and spreading of tau pathology. Neuropathol Appl Neurobiol 41(1):47-58

29. Collinge J (2016) Mammalian prions and their wider relevance in neurodegenerative diseases. Nature 539(7628):217-226 
30. Cook C et al (2014) Acetylation of the KXGS motifs in tau is a critical determinant in modulation of tau aggregation and clearance. Hum Mol Genet 23(1):104-116

31. Cowan CM, Mudher A (2013) Are tau aggregates toxic or protective in tauopathies? Front Neurol 4:114

32. Cowan CM et al (2010) Soluble hyper-phosphorylated tau causes microtubule breakdown and functionally compromises normal tau in vivo. Acta Neuropathol 120(5):593-604

33. Cowan CM et al (2015) Rescue from tau-induced neuronal dysfunction produces insoluble tau oligomers. Sci Rep 5:17191

34. Croft CL et al (2017) Membrane association and release of wild-type and pathological tau from organotypic brain slice cultures. Cell Death Dis 8(3):e2671

35. Crowther RA, Goedert M (2000) Abnormal tau-containing filaments in neurodegenerative diseases. J Struct Biol 130(2-3):271-279

36. de Calignon A et al (2012) Propagation of tau pathology in a model of early Alzheimer's disease. Neuron 73(4):685-697

37. Despres C et al (2017) Identification of the tau phosphorylation pattern that drives its aggregation. Proc Natl Acad Sci U S A 114(34):9080-9085

38. Dourlen $\mathrm{P}$ et al (2017) Functional screening of Alzheimer risk loci identifies PTK2B as an in vivo modulator and early marker of tau pathology. Mol Psychiatry 22(6):874-883

39. Dujardin S, Colin M, Buée L (2015) Invited review: animal models of tauopathies and their implications for research/translation into the clinic. Neuropathol Appl Neurobiol 41(1):59-80

40. Dujardin S et al (2014a) Neuron-to-neuron wild-type tau protein transfer through a trans-synaptic mechanism: relevance to sporadic tauopathies. Acta Neuropathol Commun 2:14

41. Dujardin S et al (2014b) Ectosomes: a new mechanism for non-exosomal secretion of tau protein. PLoS One 9(6):e100760

42. Duyckaerts C et al (1997) Dissociation of Alzheimer type pathology in a disconnected piece of cortex. Acta Neuropathol 93(5):501-507

43. Eisele YS, Duyckaerts C (2016) Propagation of Abeta pathology: hypotheses, discoveries, and yet unresolved questions from experimental and human brain studies. Acta Neuropathol 131(1):5-25

44. Espuny-Camacho I et al (2017) Hallmarks of Alzheimer's disease in stem-cell-derived human neurons transplanted into mouse brain Neuron 93(5):1066-1081 e8

45. Falcon B et al (2015) Conformation determines the seeding potencies of native and recombinant tau aggregates. J Biol Chem 290(2):1049-1065

46. Fitzpatrick AWP et al (2017) Cryo-EM structures of tau filaments from Alzheimer's disease. Nature 547(7662):185-190

47. Funk KE et al (2014) Lysine methylation is an endogenous post-translational modification of tau protein in human brain and a modulator of aggregation propensity. Biochem J 462(1):77-88

48. Furman JL et al (2017) Widespread tau seeding activity at early Braak stages. Acta Neuropathol 133(1):91-100

49. Gamblin TC et al (2003) Caspase cleavage of tau: linking amyloid and neurofibrillary tangles in Alzheimer's disease. Proc Natl Acad Sci U S A 100(17):10032-10037

50. Ghetti B et al (2015) Invited review: Frontotemporal dementia caused by microtubule-associated protein tau gene (MAPT) mutations: a chameleon for neuropathology and neuroimaging. Neuropathol Appl Neurobiol 41(1):24-46

51. Gilley J et al (2012) Age-dependent axonal transport and locomotor changes and tau hypophosphorylation in a "P301L" tau knockin mouse Neurobiol Aging 33(3):621-6e1 -621 e15

52. Gilley $\mathrm{J}$ et al (2016) Mislocalization of neuronal tau in the absence of tangle pathology in phosphomutant tau knockin mice. Neurobiol Aging 39:1-18

53. Goedert M (2016) The ordered assembly of tau is the gain-of-toxic function that causes human tauopathies. Alzheimers Dement 12(10):1040-1050

54. Goedert M, Eisenberg DS, Crowther RA (2017) Propagation of tau aggregates and neurodegeneration. Annu Rev Neurosci 40:189-210

55. Goedert M, Spillantini MG (2017) Propagation of tau aggregates. Mol Brain 10(1):18

56. Goedert $\mathrm{M}$ et al (1988) Cloning and sequencing of the CDNA encoding a core protein of the paired helical filament of Alzheimer disease: identification as the microtubule-associated protein tau. Proc Natl Acad Sc U S A 85(11):4051-4055

57. Goedert M et al (1989) Multiple isoforms of human microtubule-associated protein tau: sequences and localization in neurofibrillary tangles of Alzheimer's disease. Neuron 3(4):519-526
58. Goedert M et al (1996) Assembly of microtubule-associated protein tau into Alzheimer-like filaments induced by sulphated glycosaminoglycans. Nature 383(6600):550-553

59. Gomez-Ramos A et al (2008) Extracellular tau promotes intracellular calcium increase through M1 and M3 muscarinic receptors in neuronal cells. Mol Cell Neurosci 37(4):673-681

60. Gomez-Ramos A et al (2009) Characteristics and consequences of muscarinic receptor activation by tau protein. Eur Neuropsychopharmacol 19(10):708-717

61. Gorlovoy P et al (2009) Accumulation of tau induced in neurites by microglial proinflammatory mediators. FASEB J 23(8):2502-2513

62. Götz J et al (1995) Somatodendritic localization and hyperphosphorylation of tau protein in transgenic mice expressing the longest human brain tau isoform. EMBO J 14(7):1304-1313

63. Götz J J et al (2001) Formation of neurofibrillary tangles in P301l tau transgenic mice induced by Abeta 42 fibrils. Science 293(5534):1491-1495

64. Guo JL et al (2013) Distinct alpha-synuclein strains differentially promote tau inclusions in neurons. Cell 154(1):103-117

65. Guo JL et al (2016) Unique pathological tau conformers from Alzheimer's brains transmit tau pathology in nontransgenic mice. J Exp Med 213(12): 2635-2654

66. Guo T, Noble W, Hanger DP (2017) Roles of tau protein in health and disease. Acta Neuropathol 133(5):665-704

67. Hasegawa M et al (1996) Characterization of mAb AP422, a novel phosphorylation-dependent monoclonal antibody against tau protein. FEBS Lett 384(1):25-30

68. Hasegawa M et al (1997) Alzheimer-like changes in microtubule-associated protein tau induced by sulfated glycosaminoglycans. J Biol Chem 272(52): 33118-33124

69. Héraud C et al (2014) Increased misfolding and truncation of tau in APP/PS1/tau transgenic mice compared to mutant tau mice. Neurobiol Dis 62:100-112

70. Höglinger GU et al (2011) Identification of common variants influencing risk of the tauopathy progressive supranuclear palsy. Nat Genet 43(7):699-705

71. Holmes BB et al (2013) Heparan sulfate proteoglycans mediate internalization and propagation of specific proteopathic seeds. Proc Natl Acad Sci U S A 110(33):E3138-E3147

72. Holmes BB et al (2014) Proteopathic tau seeding predicts tauopathy in vivo. Proc Natl Acad Sci U S A 111(41):E4376-E4385

73. Hu W et al (2016) Hyperphosphorylation determines both the spread and the morphology of tau pathology. Alzheimers Dement 12(10):1066-1077

74. Hutton $\mathrm{M}$ et al (1998) Association of missense and 5'-splice-site mutations in tau with the inherited dementia FTDP-17. Nature 393(6686):702-705

75. Iba M et al (2013) Synthetic tau fibrils mediate transmission of neurofibrillary tangles in a transgenic mouse model of Alzheimer's-like tauopathy. J Neurosci 33(3):1024-1037

76. Iba M et al (2015) Tau pathology spread in PS19 tau transgenic mice following locus coeruleus (LC) injections of synthetic tau fibrils is determined by the LC's afferent and efferent connections. Acta Neuropathol 130(3):349-362

77. Irwin DJ et al (2012) Acetylated tau, a novel pathological signature in Alzheimer's disease and other tauopathies. Brain 135(Pt 3):807-818

78. Irwin DJ et al (2013) Acetylated tau neuropathology in sporadic and hereditary tauopathies. Am J Pathol 183(2):344-351

79. Jackson SJ et al (2016) Short fibrils constitute the major species of seedcompetent tau in the brains of mice transgenic for human P301S tau. J Neurosci 36(3):762-772

80. Jaunmuktane $Z$ et al (2015) Evidence for human transmission of amyloid-beta pathology and cerebral amyloid angiopathy. Nature 525(7568):247-250

81. Kampers T et al (1996) RNA stimulates aggregation of microtubuleassociated protein tau into Alzheimer-like paired helical filaments. FEBS Lett 399(3):344-349

82. Karch CM, Jeng AT, Goate AM (2012) Extracellular tau levels are influenced by variability in tau that is associated with tauopathies. J Biol Chem 287(51): 42751-42762

83. Kaufman SK et al (2016) Tau prion strains dictate patterns of cell pathology, progression rate, and regional vulnerability in vivo. Neuron 92(4):796-812

84. Kaufman SK et al (2017) Characterization of tau prion seeding activity and strains from formaldehyde-fixed tissue. Acta Neuropathol Commun 5(1):41 
85. Kovacs GG (2016) Molecular pathological classification of neurodegenerative diseases: Turning towards precision medicine. Int J Mol Sci 17(2):E189

86. Kovacs GG (2015) Invited review: neuropathology of tauopathies: principles and practice. Neuropathol Appl Neurobiol 41(1):3-23

87. Lasagna-Reeves CA et al (2011) Tau oligomers impair memory and induce synaptic and mitochondrial dysfunction in wild-type mice. Mol Neurodegener 6:39

88. Lasagna-Reeves CA et al (2012) Identification of oligomers at early stages of tau aggregation in Alzheimer's disease. FASEB J 26(5):1946-1959

89. Le Guennec, K., et al., 17q21.31 duplication causes prominent tau-related dementia with increased MAPT expression. Mol Psychiatry 22(8):1119-1125

90. Lewis J et al (2001) Enhanced neurofibrillary degeneration in transgenic mice expressing mutant tau and APP. Science 293(5534):1487-1491

91. Liu L et al (2012) Trans-synaptic spread of tau pathology in vivo. PLoS One 7(2):e31302

92. Matsuo ES et al (1994) Biopsy-derived adult human brain tau is phosphorylated at many of the same sites as Alzheimer's disease paired helical filament tau. Neuron 13(4):989-1002

93. Mirbaha $\mathrm{H}$ et al (2015) Tau trimers are the minimal propagation unit spontaneously internalized to seed intracellular aggregation. J Biol Chem 290(24):14893-14903

94. Mohamed NV, Desjardins A, Leclerc N (2017) Tau secretion is correlated to an increase of Golgi dynamics. PLoS One 12(5):e0178288

95. Moreau K et al (2014) PICALM modulates autophagy activity and tau accumulation. Nat Commun 5:4998

96. Morris M et al (2015) Tau post-translational modifications in wild-type and human amyloid precursor protein transgenic mice. Nat Neurosci 18(8):1183-1189

97. Mudher A et al (2004) GSK-3beta inhibition reverses axonal transport defects and behavioural phenotypes in Drosophila. Mol Psychiatry 9(5):522-530

98. Mudher AK et al (1999) Induction of hyperphosphorylated tau in living slices of rat hippocampal formation and subsequent detection using an ELISA. J Neurosci Methods 88(1):15-25

99. Nobuhara CK et al (2017) Tau antibody targeting pathological species blocks neuronal uptake and interneuron propagation of tau in vitro. Am J Pathol 187(6):1399-1412

100. Ozcelik S et al (2016) Co-expression of truncated and full-length tau induces severe neurotoxicity. Mol Psychiatry 21(12):1790-1798

101. Pearce $M M$ et al (2015) Prion-like transmission of neuronal huntingtin aggregates to phagocytic glia in the Drosophila brain. Nat Commun 6:6768

102. Peeraer $\mathrm{E}$ et al (2015) Intracerebral injection of preformed synthetic tau fibrils initiates widespread tauopathy and neuronal loss in the brains of tau transgenic mice. Neurobiol Dis 73:83-95

103. Perez M et al (1996) Polymerization of tau into filaments in the presence of heparin: the minimal sequence required for tau-tau interaction. J Neurochem 67(3):1183-1190

104. Planel E et al (2007) Anesthesia leads to tau hyperphosphorylation through inhibition of phosphatase activity by hypothermia. J Neurosci 27(12):3090-3097

105. Plouffe V et al (2012) Hyperphosphorylation and cleavage at D421 enhance tau secretion. PLoS One 7(5):e36873

106. Polanco JC et al (2016) Extracellular vesicles isolated from the brains of rTg4510 mice seed tau protein aggregation in a threshold-dependent manner. J Biol Chem 291(24):12445-12466

107. Pooler AM et al (2013) Physiological release of endogenous tau is stimulated by neuronal activity. EMBO Rep 14(4):389-394

108. Pooler AM et al (2015) Amyloid accelerates tau propagation and toxicity in a model of early Alzheimer's disease. Acta Neuropathol Commun 3:14

109. Reynolds MR et al (2006) Tau nitration occurs at tyrosine 29 in the fibrillar lesions of Alzheimer's disease and other tauopathies. J Neurosci 26(42):10636-10645

110. Rodriguez $L$ et al (2017) Rab7A regulates tau secretion. J Neurochem 141(4):592-605

111. Saijo E et al (2017) Ultrasensitive and selective detection of 3-repeat tau seeding activity in Pick disease brain and cerebrospinal fluid. Acta Neuropathol 133(5):751-765

112. Sanders DW et al (2014) Distinct tau prion strains propagate in cells and mice and define different tauopathies. Neuron 82(6):1271-1288

113. Sankaranarayanan $S$ et al (2015) Passive immunization with phospho-tau antibodies reduces tau pathology and functional deficits in two distinct mouse tauopathy models. PLoS One 10(5):e0125614
114. Santacruz K et al (2005) Tau suppression in a neurodegenerative mouse model improves memory function. Science 309(5733):476-481

115. Schneider A et al (1999) Phosphorylation that detaches tau protein from microtubules (Ser262, Ser214) also protects it against aggregation into Alzheimer paired helical filaments. Biochemistry 38(12):3549-3558

116. Scott JR, Davies D, Fraser H (1992) Scrapie in the central nervous system: neuroanatomical spread of infection and Sinc control of pathogenesis. J Gen Virol 73(Pt 7):1637-1644

117. Seubert $P$ et al (1995) Detection of phosphorylated Ser262 in fetal tau, adult tau, and paired helical filament tau. J Biol Chem 270(32): 18917-18922

118. Shi $Y$ et al (2017) ApoE4 markedly exacerbates tau-mediated neurodegeneration in a mouse model of tauopathy. Nature 549(7673): 523-527

119. Simon D et al (2012) Proteostasis of tau. Tau overexpression results in its secretion via membrane vesicles. FEBS Lett 586(1):47-54

120. Spillantini MG et al (1998) Mutation in the tau gene in familial multiple system tauopathy with presenile dementia. Proc Natl Acad Sci U S A 95(13):7737-7741

121. Spires-Jones TL, Attems J, Thal DR ( 2017) Interactions of pathological proteins in neurodegenerative diseases. Acta Neuropathol 134(2):187-205

122. Spires-Jones TL et al (2011) Are tangles as toxic as they look? J Mol Neurosci 45(3):438-444

123. Spittaels K et al (1999) Prominent axonopathy in the brain and spinal cord of transgenic mice overexpressing four-repeat human tau protein. Am $J$ Pathol 155(6):2153-2165

124. Stancu IC et al (2015) Templated misfolding of tau by prion-like seeding along neuronal connections impairs neuronal network function and associated behavioral outcomes in tau transgenic mice. Acta Neuropathol 129(6):875-894

125. Takahashi $M$ et al (2015) Extracellular association of APP and tau fibrils induces intracellular aggregate formation of tau. Acta Neuropathol 129(6):895-907

126. Takeda S et al (2016) Seed-competent high-molecular-weight tau species accumulates in the cerebrospinal fluid of Alzheimer's disease mouse model and human patients. Ann Neurol 80(3):355-367

127. Taniguchi-Watanabe $S$ et al (2016) Biochemical classification of tauopathies by immunoblot, protein sequence and mass spectrometric analyses of sarkosylinsoluble and trypsin-resistant tau. Acta Neuropathol 131(2):267-280

128. Tardivel $M$ et al (2016) Tunneling nanotube (TNT)-mediated neuron-to neuron transfer of pathological tau protein assemblies. Acta Neuropathol Commun 4(1):117

129. Thal DR et al (2002) Phases of Abeta-deposition in the human brain and its relevance for the development of AD. Neurology 58(12):1791-1800

130. Thomas SN et al (2012) Dual modification of Alzheimer's disease PHF-tau protein by lysine methylation and ubiquitylation: a mass spectrometry approach. Acta Neuropathol 123(1):105-117

131. Uchihara T (2007) Silver diagnosis in neuropathology: principles, practice and revised interpretation. Acta Neuropathol 113(5):483-499

132. Van Cauwenberghe C, Van Broeckhoven C, Sleegers K (2016) The genetic landscape of Alzheimer disease: clinical implications and perspectives. Genet Med 18(5):421-430

133. Vasconcelos B et al (2016) Heterotypic seeding of tau fibrillization by pre-aggregated Abeta provides potent seeds for prion-like seeding and propagation of tau-pathology in vivo. Acta Neuropathol 131:549

134. von Bergen M et al (2000) Assembly of tau protein into Alzheimer paired helical filaments depends on a local sequence motif ((306)VQIVYK(311)) forming beta structure. Proc Natl Acad Sci U S A 97(10):5129-5134

135. Walsh DM, Selkoe DJ (2016) A critical appraisal of the pathogenic protein spread hypothesis of neurodegeneration. Nat Rev Neurosci 17(4):251-260

136. Wang $Y$ et al (2017) The release and trans-synaptic transmission of tau via exosomes. Mol Neurodegener 12(1):5

137. Wegmann S et al (2015) Removing endogenous tau does not prevent tau propagation yet reduces its neurotoxicity. EMBO J 34(24):3028-3041

138. Wilson DM, Binder LI (1997) Free fatty acids stimulate the polymerization of tau and amyloid beta peptides. In vitro evidence for a common effector of pathogenesis in Alzheimer's disease. Am J Pathol 150(6):2181-2195

139. Wischik CM et al (1988) Structural characterization of the core of the paired helical filament of Alzheimer disease. Proc Natl Acad Sci U S A 85(13):4884-4888 
140. Wittmann CW et al (2001) Tauopathy in Drosophila: neurodegeneration without neurofibrillary tangles. Science 293(5530):711-714

141. Wray $S$ et al (2008) Direct analysis of tau from PSP brain identifies new phosphorylation sites and a major fragment of N-terminally cleaved tau containing four microtubule-binding repeats. J Neurochem 105(6):2343-2352

142. Wu JW et al (2016) Neuronal activity enhances tau propagation and tau pathology in vivo. Nat Neurosci 19(8):1085-1092

143. Yamada K et al (2011) In vivo microdialysis reveals age-dependent decrease of brain interstitial fluid tau levels in P301S human tau transgenic mice. J Neurosci 31(37):13110-13117

144. Yamada K et al (2014) Neuronal activity regulates extracellular tau in vivo. J Exp Med 211(3):387-393

145. Yanamandra K et al (2013) Anti-tau antibodies that block tau aggregate seeding in vitro markedly decrease pathology and improve cognition in vivo. Neuron 80(2):402-414

146. Yetman MJ et al (2016) Transgene expression in the Nop-tTA driver line is not inherently restricted to the entorhinal cortex. Brain Struct Funct 221(4): 2231-2249

147. Zhang $X$ et al (2017) RNA stores tau reversibly in complex coacervates. PLoS Biol 15(7):e2002183

148. Zhao $\mathrm{N}$ et al (2017) Apolipoprotein E4 impairs neuronal insulin signaling by trapping insulin receptor in the endosomes. Neuron 96(1):115-129 e5

149. Zhong Q et al (2012) Tau isoform composition influences rate and extent of filament formation. J Biol Chem 287(24):20711-20719

150. Zilka N et al (2012) The self-perpetuating tau truncation circle. Biochem Soc Trans 40(4):681-686

\section{Submit your next manuscript to BioMed Central and we will help you at every step:}

- We accept pre-submission inquiries

- Our selector tool helps you to find the most relevant journal

- We provide round the clock customer support

- Convenient online submission

- Thorough peer review

- Inclusion in PubMed and all major indexing services

- Maximum visibility for your research

Submit your manuscript at www.biomedcentral.com/submit 